\title{
(6) OPEN ACCESS \\ Diagnostic accuracy of ultrasonography, MRI and MR arthrography in the characterisation of rotator cuff disorders: a systematic review and meta-analysis
}

\author{
Jean-Sébastien Roy, ${ }^{1,2}$ Caroline Braën, ${ }^{2,3}$ Jean Leblond, ${ }^{2}$ François Desmeules, ${ }^{3,4}$ \\ Clermont E Dionne, ${ }^{1,5}$ Joy C MacDermid, ${ }^{6}$ Nathalie J Bureau, ${ }^{7}$ Pierre Frémont ${ }^{1,5}$
}

\begin{abstract}
- Additional material is published online only. To view please visit the journal online (http://dx.doi.org/10.1136/ bjsports-2014-094148)

For numbered affiliations see end of article.
\end{abstract}

\section{Correspondence to} Dr Jean-Sébastien Roy, Centre interdisciplinaire de recherche en réadaptation et en intégration sociale (CIRRIS), 525, boulevard Wilfrid-Hamel, local H-1710, Quebec, Canada G1M 2S8: jean-sebastien.roy@rea.ulaval.ca

Received 15 August 2014 Revised 9 January 2015 Accepted 14 January 2015 Published Online First 12 February 2015

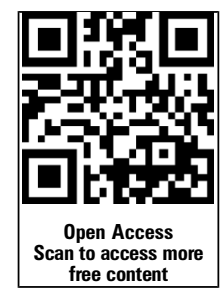

CrossMark

To cite: Roy J-S, Braën C, Leblond J, et al. Br J Sports Med 2015;49:1316-1328.

\section{ABSTRACT}

Background Different diagnostic imaging modalities, such as ultrasonography (US), MRI, MR arthrography (MRA) are commonly used for the characterisation of rotator cuff (RC) disorders. Since the most recent systematic reviews on medical imaging, multiple diagnostic studies have been published, most using more advanced technological characteristics. The first objective was to perform a meta-analysis on the diagnostic accuracy of medical imaging for characterisation of RC disorders. Since US is used at the point of care in environments such as sports medicine, a secondary analysis assessed accuracy by radiologists and nonradiologists.

Methods A systematic search in three databases was conducted. Two raters performed data extraction and evaluation of risk of bias independently, and agreement was achieved by consensus. Hierarchical summary receiver-operating characteristic package was used to calculate pooled estimates of included diagnostic studies. Results Diagnostic accuracy of US, MRI and MRA in the characterisation of full-thickness RC tears was high with overall estimates of sensitivity and specificity over 0.90 . As for partial RC tears and tendinopathy, overall estimates of specificity were also high $(>0.90)$, while sensitivity was lower (0.67-0.83). Diagnostic accuracy of US was similar whether a trained radiologist,

sonographer or orthopaedist performed it.

Conclusions Our results show the diagnostic accuracy of US, MRI and MRA in the characterisation of fullthickness RC tears. Since full thickness tear constitutes a key consideration for surgical repair, this is an important characteristic when selecting an imaging modality for RC disorder. When considering accuracy, cost, and safety, US is the best option.

\section{INTRODUCTION}

Shoulder pathology is common and associated with substantial functional limitations that increase with age. ${ }^{1}$ Disorders of the rotator cuff (RC) tendons constitute the most common group of pathologies of the shoulder. ${ }^{2} \mathrm{RC}$ disorders are of multifactorial origin and may result in a progressive degeneration of the RC tendons. ${ }^{3}$ Knowledge of the integrity of the RC tendons in an episode of subacromial pain is an important factor to consider in treatment decision-making (surgical vs conservative management) ${ }^{4}$ and clinicians use a variety of diagnostic tests to detect RC disorders and to define the extent of damage. Medical imaging technologies, such as ultrasonography (US), MRI and MR arthrography (MRA) provide clinically useful information that can help establish the proper treatment plan. ${ }^{5}$

Several systematic reviews have been published on the diagnostic accuracy of medical imaging for the characterisation of RC disorders. ${ }^{5-11}$ However, since the publication of the last systematic reviews, ${ }^{5} 1011$ more than 10 diagnostic studies, mostly with sound methodology, have been published, most of them using more advance technology and technique, such as devices with higher frequency transducers for US and higher field strength magnets for MRA and MRI. Also, a recent statistical package, the hierarchical summary receiver-operating characteristic (HSROC) has been proposed to optimise the realisation of meta-analyses on the accuracy of diagnostic tests. ${ }^{12}$ This statistical approach enables the calculation of pooled estimates of the sensitivity and specificity of a test and provides a mechanism to provide more precise estimates of the diagnostic accuracy of different imaging methods.

When new data are available, as it is currently, a meta-analysis is warranted to determine if these new data have an impact on the diagnostic accuracy of a given tool. Especially since technology and technique have been improving over the years for imaging modalities, and that a recent statistical package has been shown to optimise meta-analyses.

Another aspect of musculoskeletal imaging modalities that have changed recently is the increase use of US at the point of care in environments such as sport medicine. ${ }^{13}$ In fact, nonradiologists such as specialists in physical medicine, rheumatology, orthopaedic and family medicine are currently using US during physical examinations. ${ }^{14}$ However, given the user-dependent nature of US, health professionals must make sure that they possess the competence and training before using it. Therefore, a synthesis of the literature is also needed to determine if the diagnostic accuracy of US is similar when non-radiologists and radiologists use this technology.

The primary objective of this study was to perform a systematic review with a meta-analysis on the diagnostic accuracy of US, MRI and MRA for the characterisation of tendinopathy, partial thickness RC tears and full-thickness RC tears in individuals with shoulder pain. Secondary objectives were to compare the accuracy of these imaging modalities depending on the inclusion criteria of participants in the studies, as well as regarding the technological characteristics of the 
equipment used in the included studies. Finally, since US is used at the point of care, another secondary analysis was to assess the diagnostic accuracy by radiologists and non-radiologists.

\section{METHODS}

The presentation of this systematic review follows recommendations of PRISMA. ${ }^{15}$

\section{Search methods for eligible studies}

Searches for eligible articles were conducted in Medline, Embase and CINHAL databases from their date of inception to December 2013 using the following search strategy:

\begin{abstract}
«Shoulder joint» $[\mathrm{MeSH}] \mathrm{OR}$ "Shoulder impingement syndrome » $\mathrm{OR}$ « Subacromial impingement » $\mathrm{OR}$ «Rotator cuff» $[\mathrm{MeSH}]$ OR ((Bursitis[MeSH] OR Tendinopathy[MeSH]) AND shoulder) $\mathrm{OR}$ «Shoulder pain» $[\mathrm{MeSH}]$ OR Shoulder
\end{abstract}

\section{AND}

"Diagnostic imaging "[MeSH] OR Ultrasound OR Ultrasono* OR Sonograph* OR MRI OR " magnetic resonance imaging " OR MRA OR « magnetic resonance arthrography »

\section{AND}

«Sensitivity and specificity»[MeSH] OR «Diagnostic accuracy» OR «Diagnostic value» OR Accuracy OR Reliability OR « diagnostic efficiency»

This search strategy was adapted to each database. Reference lists of retrieved studies and previous systematic reviews were also searched to identify additional relevant publications.

\section{Eligibility criteria}

Articles were included if they met the following inclusion criteria: (1) included adult participants with shoulder pain; (2) used MRI, MRA or US as index test, and surgery (arthroscopy or open surgery) as reference standard; (3) reported on diagnostic accuracy of medical imaging for the characterisation of an RC disorder (tendinitis/tendinosis/tendinopathy (subacromial impingement syndrome), full or partial RC tears). For the purpose of this study, all imaging findings used in the selected studies to qualify RC abnormalities other than RC tears will be referred to as 'tendinopathy'. For the meta-analysis, only articles that published a $2 \times 2$ table or included data that allowed the construction of a $2 \times 2$ table were included.

\section{Data extraction and risk of bias evaluation}

Data were extracted for participants' characteristics, index test used including specific equipment's characteristics, reference standard (who administered the tests, time between the tests). Data on diagnostic accuracy were also extracted. A first reader extracted the data. A second reader then corroborated or completed it if data were missing.

The risk of bias was evaluated for each article with the QUADAS 2 (Quality Assessment Tool for Diagnostic Accuracy Studies) that evaluates four domains that may bias the results. ${ }^{16}$ The first one is Patient selection (participants recruited consecutively or selected randomly). Participants included in the study had to represent a large spectrum of conditions that included the target condition, but was not restrained to it. Thus, studies were scored as having a high risk of bias when only participants strongly suspected of having RC disorders were considered. The second domain relates to bias that could be introduced by the Index test (blinding when interpreting the index test, clear description of the diagnostic thresholds). The third domain refers to the Reference standard or 'gold standard' (as near as possible to perfect accuracy, interpreted without the results of the index test). The last domain is the Flow and timing (patient retention, time period between index test and reference standard). A study was scored as having a high risk of bias for this domain when the interval between the index test and the reference standard was more than 30 days. ${ }^{17}$ Two raters independently evaluated each article with the QUADAS 2. After the independent evaluation, the pair of raters met to discuss the article. Each specific domain was openly discussed to reach consensus. A preconcensus inter-rater agreement was calculated for each specific domain with the $\kappa$ statistic.

\section{Statistical analyses}

Data were analysed with V.3.0.2 of the R statistical software (http://www.r-project.org/). The HSROC package was used to calculate overall pooled estimates of the included diagnostic studies by taking into account the between-study and withinstudy variability. ${ }^{12}$ This routine, based on Bayesian rules, estimates the overall sensitivity $(\mathrm{Sn})$ and specificity $(\mathrm{Sp})$ for a group of studies and produces a ROC curve with confidence and credible intervals. The classical confidence interval (CI) presumes that differences in $\mathrm{Sn}$ and $\mathrm{Sp}$ between studies are caused only by a statistical instability related to sampling or measurement errors. All estimates would turn around a unique value of $\mathrm{Sn}$ and a unique value of $\mathrm{Sp}$. In reality, for a same technique, $\mathrm{Sn}$ and $\mathrm{Sp}$ may vary in time, with different populations, with different operators or any other relevant conditions that change the nature of the test. Across different conditions, Sn and Sp could fluctuate among a range of values that reflect a change in reality rather than a statistical instability. The credible intervals delimit how the reality of $\mathrm{Sn}$ and $\mathrm{Sp}$ could fluctuate for reasons other than sampling or measurement errors. In this context, the CI adds to the credible interval the uncertainty caused by the sampling and measurement errors. The credible interval lies within the CI. Heterogeneity was explored graphically with forest plot. Positive $(\mathrm{LR}+)$ and negative (LR-) likelihood ratios were calculated from the overall $\mathrm{Sn}$ and $\mathrm{Sp} .{ }^{18}$ However, confidence and credible intervals could not be calculated for likelihood ratios.

Data on diagnostic accuracy of MRI, MRA and US were pooled for (1) full or partial RC tears, (2) full RC tears only, (3) partial RC tears only and (4) tendinopathy. Thereafter, for each of these categories, meta-analyses were performed for studies with a high risk of bias for the participant selection domain of the QUADAS 2 and studies with a low risk of bias on the same domain. Meta-analyses were also performed for equipment characteristics (US frequency $\leq 7.5 \mathrm{MHz}$ or $>7.5 \mathrm{MHz}$; MRI field strength $\leq 1.5 \mathrm{~T}$ or $3 \mathrm{~T}$ ) and regarding the professionals who performed the index test (only for US studies since MRI and MRA are interpreted by radiologists in the included studies).

\section{RESULTS}

\section{Search results and selected articles}

Searches resulted in 2304 citations (duplicates removed). Two evaluators screened the titles and abstracts for eligible articles. Two hundred and sixty-four articles were accepted for full review, while 2040 were excluded by consensus. In addition, eight articles were obtained from reference list of selected results and previous reviews, for a total of 272 articles eligible for full review. Of these articles, 82 reached eligibility for meta-analysis (47 for US, 29 for MRI and 21 for MRA; figure 1, see online supplementary appendix 1). 
Figure 1 Flow diagram of the bibliographic search.
2304 (duplicates removed) located citations (PubMed, Embase, CINAHL)

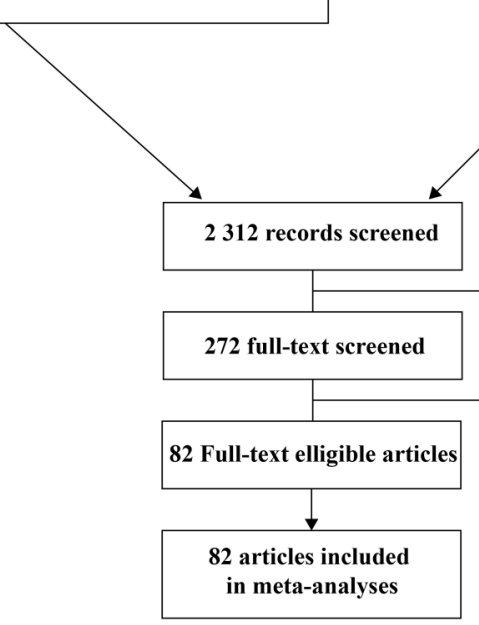

additional records by handsearching

2040 records excluded

190 articles excluded for: Population: 22 Incomplete data reported: 47 Reviews: 16

Not a diagnostic accuracy study: 48 Use of another index test: 18

Use of another reference standard: 39

\section{Risk of bias assessment of the included studies}

For the risk of bias evaluation, the two raters were fairly concordant, with average $\kappa$ inter-rater ranging from 0.33 to 0.64 .

The evaluation of the risk of bias shows recurrent sources of bias on three of the four items of the QUADAS 2 tool (figure 2). The first item, participant selection, was rated as a high risk of bias in $61 \%$ of the studies; meaning that in most studies, a preselection of participants was made. The third item, related to the reference standard, has also been frequently rated as a high risk of bias given that the surgeons were often aware of the results of imaging tests before the surgery. Considering the invasive nature of the reference standard, this is understandable as it would have

\section{A Studies on ultrasonography accuracy}

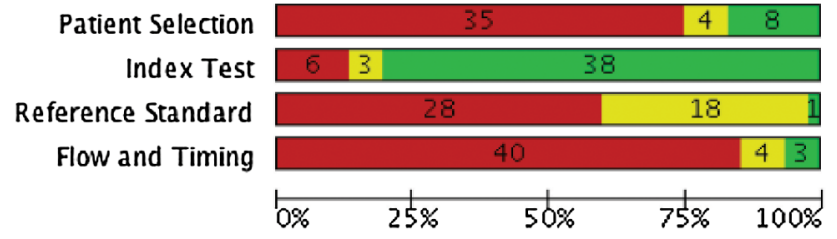

\section{B Studies on magnetic resonance imaging accuracy}

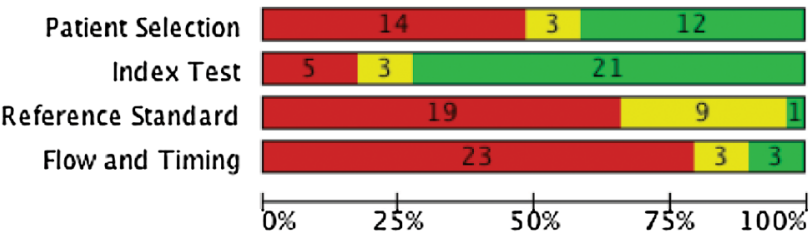

\section{Studies on magnetic resonance arthrography accuracy}

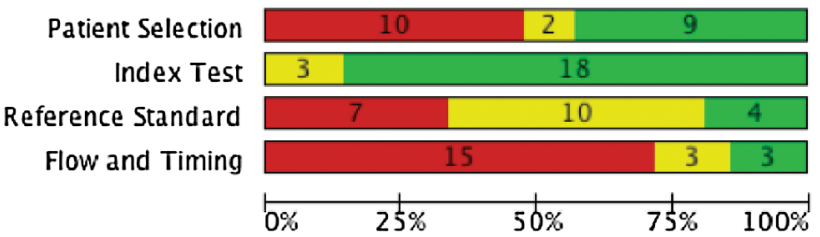

Figure 2 Risk of bias assessment of included studies using the QUADAS 2 tool. For the 3 imaging modalities $(A-C)$, each bar shows the number and percentage of studies with a high (red), intermediate (yellow) and low (green) risk of bias for the 4 categories of QUADAS 2 tool. been unethical for surgeons to operate blindly as to the condition of the patient. The last item, flow and timing, has also often been evaluated as a high risk of bias. This is due to the fact that in most studies, the interval of time between the index test and the reference standard exceeded 30 days. The second item of the QUADAS was the one with the lowest risk of bias given that a clear description of the diagnostic threshold and of the standardised procedure for the index test was provided.

\section{Diagnostic accuracy of imaging modalities for any type \\ of RC tears}

Pooled data for all included studies

Results indicate an equivalent performance of the three imaging modalities (US, MRI and MRA) in the diagnosis of full or partial RC tears considered together (Sn: 0.90 to 0.91 ; Sp: 0.86 to 0.90 ; table 1A; figure 3). The credible intervals of the overall estimates of Sn and Sp of the MRA were larger than those of MRI and US, probably because of the smaller number of studies that evaluated MRA.

Secondary analysis based on the risk of bias in patient selection The overall Sn of the group of studies with a high risk of bias in patient selection was substantially higher than the group of studies with a low risk for MRA (Sn: 0.93 and 0.83, respectively) and US (Sn: 0.93 and 0.76, respectively; table 1B). For MRA, the $\mathrm{Sp}$ analyses show that the group of studies with a high risk of bias has a substantially higher value compared to the low risk of bias studies (Sp: 0.95 and 0.73, respectively).

\section{Secondary analysis regarding equipment characteristics}

In most studies on the diagnostic accuracy of US, a transducer frequency equal or lower to $7.5 \mathrm{MHz}$ was used, while 12 studies used a transducer frequency higher than $7.5 \mathrm{MHz}$ (table 1C). The diagnostic accuracy based on the transducer frequency used was similar for the overall $\mathrm{Sn}$ or $\mathrm{Sp}$.

In MRI studies, a $1.5 \mathrm{~T}$ or less density was mostly used, while two studies used a density of $3.0 \mathrm{~T}$. Despite the wide credible intervals for the 3.0 T studies, overall $\mathrm{Sn}$ and $\mathrm{Sp}$ of both groups were similar. This was also true for MRA studies.

Secondary analysis regarding the specialist performing the US examination

The meta-analyses showed a similar diagnostic accuracy of US when the examination was performed by a trained radiologist or 
Table 1 Overall Sn and Sp of US, MRI and MRA in the characterisation of full-thickness or partial-thickness RC tears

\begin{tabular}{|c|c|c|c|c|c|c|}
\hline & \multicolumn{6}{|c|}{ (A) Full or partial rotator cuff tears } \\
\hline & N (studies) & N (shoulders) & Sn (Cl 95\%) & Sp (CI 95\%) & LR+ & LR- \\
\hline US & $35^{\mathrm{a}}$ & 2774 & 0.91 (0.86 to 0.95$)$ & 0.86 (0.80 to 0.92$)$ & 6.5 & 0.1 \\
\hline MRI & $21^{b}$ & 1575 & $0.90(0.84$ to 0.96$)$ & $0.90(0.84$ to 0.95$)$ & 9.0 & 0.1 \\
\hline MRA & $14^{c}$ & 979 & $0.90(0.79$ to 0.97$)$ & $0.90(0.79$ to 0.97$)$ & 9.0 & 0.1 \\
\hline
\end{tabular}

\begin{tabular}{|c|c|c|c|c|c|c|c|c|c|c|c|c|}
\hline & \multicolumn{12}{|c|}{ (B) Secondary analysis based on risk of bias } \\
\hline & \multicolumn{6}{|c|}{ Studies with low risk of bias in patient selection } & \multicolumn{6}{|c|}{ Studies with high risk of bias in patient selection } \\
\hline & N (studies) & N (shoulders) & Sn (Cl 95\%) & $\mathrm{Sp}(\mathrm{Cl} 95 \%)$ & LR+ & LR- & N (studies) & $\mathrm{N}$ (shoulders) & Sn (Cl 95\%) & $\mathrm{Sp}(\mathrm{Cl} 95 \%)$ & LR+ & LR- \\
\hline US & $7^{d}$ & 552 & 0.76 (0.59 to 0.90$)$ & 0.90 (0.80 to 0.98$)$ & 7.6 & 0.3 & $24^{9}$ & 1749 & 0.93 (0.88 to 0.97$)$ & 0.84 (0.76 to 0.91$)$ & 5.8 & 0.1 \\
\hline MRI & $10^{\mathrm{e}}$ & 685 & 0.91 (0.82 to 0.97$)$ & 0.89 (0.78 to 0.97$)$ & 8.3 & 0.1 & $8^{\mathrm{h}}$ & 467 & $0.92(0.76$ to 1$)$ & $0.88(0.74$ to 0.97$)$ & 7.8 & 0.1 \\
\hline MRA & $7^{f}$ & 556 & $0.83(0.66$ to 0.96$)$ & $0.95(0.86$ to 1.0$)$ & 16.6 & 0.2 & $6^{\mathrm{i}}$ & 355 & $0.93(0.78$ to 1.0$)$ & $0.73(0.44$ to 0.94$)$ & 3.4 & 0.1 \\
\hline
\end{tabular}

\begin{tabular}{|c|c|c|c|c|c|c|c|c|c|c|c|c|}
\hline & \multicolumn{6}{|c|}{$\leq 7.5 \mathrm{MHz}$ (US), $\leq 1.5 \mathrm{~T}$ (MRI and MRA) } & \multicolumn{6}{|c|}{$>7.5 \mathrm{MHz}$ (US), 3.0 T (MRI and MRA) } \\
\hline & $\mathrm{N}$ (studies) & $\mathrm{N}$ (shoulders) & $\mathrm{Sn}(\mathrm{Cl} 95 \%)$ & $\mathrm{Sp}(\mathrm{Cl} 95 \%)$ & LR+ & LR- & N (studies) & $\mathrm{N}$ (shoulders) & Sn (CI 95\%) & Sp (CI 95\%) & LR+ & LR- \\
\hline US & $22^{\mathrm{j}}$ & 1912 & 0.91 (0.86 to 0.96$)$ & 0.87 (0.80 to 0.93$)$ & 7.0 & 0.1 & $12^{\mathrm{m}}$ & 830 & 0.93 (0.84 to 1$)$ & 0.85 (0.70 to 0.96$)$ & 6.2 & 0.1 \\
\hline MRI & $17^{\mathrm{k}}$ & 1243 & $0.89(0.81$ to 0.95$)$ & 0.89 (0.82 to 0.95$)$ & 8.1 & 0.1 & $2^{\mathrm{n}}$ & 198 & $0.81(0.45$ to 1$)$ & $0.94(0.58$ to 1$)$ & 13.5 & 0.2 \\
\hline \multirow[t]{4}{*}{ MRA } & $9^{\prime}$ & 540 & $0.86(0.72$ to 0.97$)$ & $0.86(0.66$ to 0.98$)$ & 6.1 & 0.2 & $5^{\circ}$ & 439 & 0.89 (0.64 to 1$)$ & 0.91 (0.70 to 1$)$ & 9.9 & 0.1 \\
\hline & \multicolumn{12}{|c|}{ (D) Secondary analysis based on the specialist who performed the US examination } \\
\hline & \multicolumn{6}{|l|}{ Radiologists } & \multicolumn{6}{|c|}{ Sonographers and orthopaedists } \\
\hline & N (studies) & N (shoulders) & Sn (Cl 95\%) & $\mathrm{Sp}(\mathrm{Cl} 95 \%)$ & $\mathbf{L R +}$ & LR- & N (studies) & N (shoulders) & $\mathrm{Sn}(\mathrm{Cl} 95 \%)$ & $\mathrm{Sp}(\mathrm{Cl} 95 \%)$ & LR+ & LR- \\
\hline US & $18^{\mathrm{p}}$ & 1333 & 0.89 (0.81 to 0.96$)$ & 0.85 (0.76 to 0.93$)$ & 5.9 & 0.1 & $9^{9}$ & 861 & 0.88 (0.74 to 0.98$)$ & 0.89 (0.80 to 0.96$)$ & 8 & 0.1 \\
\hline
\end{tabular}


Figure 3 Accuracy of individual studies for ultrasonography, MRI and MR arthrography in characterisation of any tear. TP, true positive; $\mathrm{FP}$, false positive; $\mathrm{FN}$, false negative; TN, true negative; $95 \% \mathrm{Cl}, 95 \%$ confidence interval.

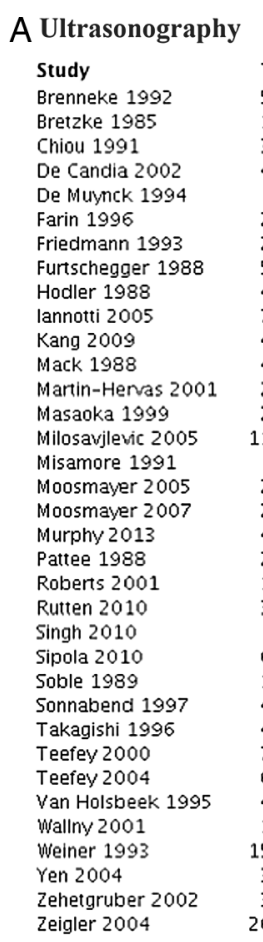

TP FP FN TN Sensitivity $(95 \% \mathrm{Cl})$ Specificity $(95 \% \mathrm{Cl})$ Sensitivity $(95 \% \mathrm{Cl})$ Specificity $(95 \% \mathrm{Cl})$ $\begin{array}{llllll}51 & 10 & 14 & 45 & 0.78[0.67,0.88] & 0.82[0.69,0.91]\end{array}$ $\begin{array}{llllllll}17 & 1 & 0 & 1 & 1.00 & 0.80,1.00] & 0.50[0.01,0.99]\end{array}$

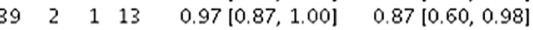
$\begin{array}{lllllll}41 & 0 & 4 & 26 & 0.91[0.79,0.98] & 1.00[0.87,1.00]\end{array}$ $\begin{array}{lllrlll}1 & 0 & 0 & 4 & 1.00[0.03,1.00] & 1.00[0.40,1.00]\end{array}$ $\begin{array}{lllllll}26 & 1 & 4 & 55 & 0.87 & {[0.69,0.96]} & 0.98[0.90,1.00]\end{array}$ $\begin{array}{llllll}25 & 0 & 6 & 15 & 0.81[0.63,0.93] & 1.00[0.78,1.00]\end{array}$ $\begin{array}{llllll}58 & 3 & 6 & 1 & 0.91[0.81,0.96] & 0.25[0.01,0.81]\end{array}$ $\begin{array}{llllll}42 & 2 & 0 & 7 & 1.00[0.92,1.00] & 0.78[0.40,0.97]\end{array}$ $\begin{array}{lllllll}76 & 4 & 3 & 16 & 0.96[0.89,0.99] & 0.80[0.56,0.94]\end{array}$ 430030 $\begin{array}{rrrr}43 & 0 & 2 & 5 \\ 43 & 1 & 4 & 42\end{array}$ $\begin{array}{llrr}43 & 1 & 4 & 42 \\ 24 & 9 & 10 & 18\end{array}$ $\begin{array}{rrrr}24 & 9 & 10 & 18 \\ 28 & 2 & 0 & 34\end{array}$ $\begin{array}{llll}18 & 4 & 6 & 62\end{array}$ $\begin{array}{llll}9 & 2 & 18 & 3\end{array}$ $\begin{array}{llll}22 & 1 & 11 & 45\end{array}$ $\begin{array}{llll}25 & 1 & 13 & 19\end{array}$ $49 \begin{array}{llll}4 & 14 & 47\end{array}$ $\begin{array}{rrrr}47 & 6 & 8 & 11\end{array}$ $\begin{array}{lllr}3 & 0 & 4 & 7\end{array}$ $\begin{array}{llll}31 & 13 & 0 & 24\end{array}$ $\begin{array}{llll}9 & 5 & 0 & 1\end{array}$ $\begin{array}{llll}61 & 6 & 5 & 5\end{array}$ $\begin{array}{llll}14 & 4 & 1 & 11\end{array}$ $\begin{array}{llll}48 & 2 & 21 & 39\end{array}$ $\begin{array}{llll}48 & 2 & 21 & 39 \\ 42 & 7 & 16 & 57\end{array}$ $\begin{array}{rrrr}42 & 7 & 16 & 57 \\ 75 & 3 & 5 & 17\end{array}$ $\begin{array}{rrrr}75 & 3 & 5 & 17 \\ 63 & 2 & 2 & 4\end{array}$ $\begin{array}{rrrr}63 & 2 & 2 & 4 \\ 48 & 3 & 1 & 15\end{array}$ $\begin{array}{llll}48 & 3 & 1 & 15\end{array}$ $\begin{array}{llll}17 & 3 & 6 & 14\end{array}$ $\begin{array}{llll}50 & 4 & 8 & 63\end{array}$ $\begin{array}{llll}38 & 1 & 2 & 9\end{array}$ $\begin{array}{llll}34 & 9 & 5 & 43\end{array}$ $0.96[0.85,0.99]$ $0.91[0.80,0.98]$ $0.71[0.53,0.85]$ $1.00[0.88,1.00]$ $0.95[0.90,0.98]$ $0.33[0.17,0.54]$ $0.67[0.48,0.82]$ $0.66[0.49,0.80]$ $0.78[0.66,0.87]$ $0.77[0.60,0.90]$ $0.76[0.50,0.93]$ $1.00[0.89,1.00]$ $1.00[0.66,1.00]$ $0.92[0.83,0.97]$ $0.93[0.68,1.00]$ $0.70[0.57,0.80]$ $0.72[0.59,0.83]$ $0.94[0.86,0.98]$ $0.97[0.89,1.00]$ $0.98[0.89,1.00]$ $0.74[0.52,0.90]$ $0.95[0.90,0.98]$ $0.95[0.83,0.99]$ $0.87[0.73,0.96]$ $0.87[0.73,0.96]$
$1.00[0.98,1.00]$ $1.00[0.48,1.00]$ $0.98[0.88,1.00]$ $0.67[0.46,0.83]$ $0.94[0.81,0.99]$ $0.94[0.85,0.98]$ $0.60[0.15,0.95]$ $0.98[0.88,1.00]$ $0.95[0.75,1.00]$ $0.84[0.72,0.92]$ $0.65[0.38,0.86]$ $1.00[0.59,1.00]$ $0.65[0.47,0.80]$ $0.17[0.00,0.64]$ $0.45[0.17,0.77]$ $0.73[0.45,0.92]$ $0.95[0.83,0.99]$ $0.89[0.79,0.95]$ $0.85[0.62,0.97]$ $0.67[0.22,0.96]$ $0.83[0.59,0.96]$ $0.82[0.57,0.96]$ $0.94[0.85,0.98]$ $0.90[0.55,1.00]$ $0.83[0.70,0.92]$ $0.86[0.42,1.00]$

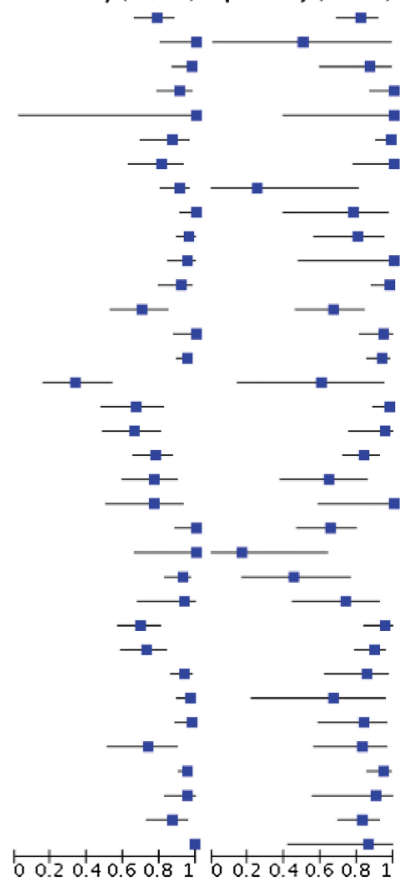

$B$ Magnetic resonance imaging

Study
Balich 1997
Frei 2008
Gagey 1993
Hitachi 2011
Hodler 1992
lannotti 2005
Karzel 1993
Lambert 2009
Magee 2009
Martin-Hervas 2001
Ostor 2013
Quinn 1995
Shellock 2001
Teefey 2004
Traughber 1992
Tuite 1994
Wang 1994
Whorowski 1997
Yagci 2001
Yamakawa 2001
Zlatkin 2004

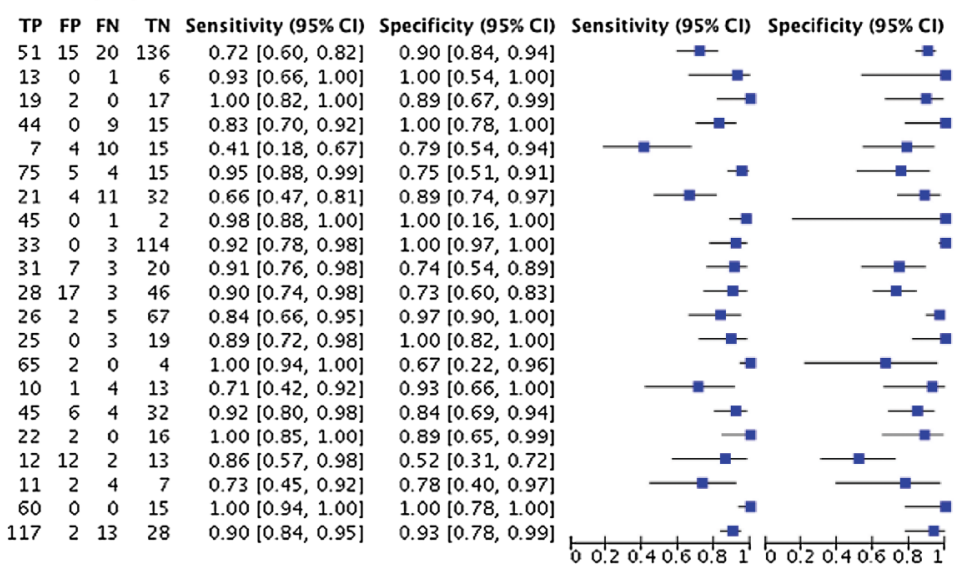

CMagnetic resonance arthrography

\begin{tabular}{|c|c|c|c|c|c|c|c|c|}
\hline Study & TP & FP & FN & TN & Sensitivity $(95 \% \mathrm{Cl})$ & Specificity (95\% CI) & Sensitivity $(95 \% \mathrm{Cl})$ & Specificity $(95 \% \mathrm{Cl})$ \\
\hline Choo 2012 & 34 & 1 & 1 & 13 & $0.97[0.85,1.00]$ & $0.93[0.66,1.00]$ & $\rightarrow$ & \\
\hline Herold 2006 & 31 & 1 & 3 & 16 & $0.91[0.76,0.98]$ & $0.94[0.71,1.00]$ & $\rightarrow$ & \\
\hline Hitachi 2011 & 15 & 0 & 2 & 11 & $0.88[0.64,0.99]$ & $1.00[0.72,1.00]$ & $\longrightarrow$ & \\
\hline Hodler 1992 & 12 & 3 & 5 & 16 & $0.71[0.44,0.90]$ & $0.84[0.60,0.97]$ & 上 & \\
\hline Jung 2012 & 58 & 2 & 3 & 24 & $0.95[0.86,0.99]$ & $0.92[0.75,0.99]$ & & \\
\hline Kang 2009 & 45 & 1 & 0 & 4 & $1.00[0.92,1.00]$ & $0.80[0.28,0.99]$ & $\rightarrow$ & \\
\hline Karzel 1993 & 26 & 2 & 6 & 34 & $0.81[0.64,0.93]$ & $0.94[0.81,0.99]$ & & \\
\hline magee 2009 & 36 & 0 & 0 & 114 & $1.00[0.90,1.00]$ & $1.00[0.97,1.00]$ & $\rightarrow$ & - \\
\hline Hodi 2013 & 8 & 2 & 6 & 87 & $0.57[0.29,0.82]$ & $0.98[0.92,1.00]$ & {[} & \\
\hline Mohtadi 2004 & 26 & 21 & 10 & 1 & $0.72[0.55,0.86]$ & $0.05[0.00,0.23]$ & $\longrightarrow-$ & $=$ \\
\hline Schreinemachers 2009 & 13 & 7 & 10 & 62 & $0.57[0.34,0.77]$ & $0.90[0.80,0.96]$ & & $\rightarrow$ \\
\hline Sipola 2010 & 62 & 2 & 2 & 9 & $0.97[0.89,1.00]$ & $0.82[0.48,0.98]$ & $\rightarrow$ & - \\
\hline Stoppino 2013 & 33 & 1 & 3 & 71 & $0.92[0.78,0.98]$ & $0.99[0.93,1.00]$ & $\rightarrow$ & \\
\hline Yagci 2001 & 15 & 2 & 0 & 7 & $1.00[0.78,1.00]$ & $0.78[0.40,0.97]$ & & \\
\hline
\end{tabular}

by other professionals (trained sonographers or orthopaedists; table 1D).

\section{Diagnostic accuracy of imaging modalities for full-thickness RC tears}

Pooled data for all included studies

For the diagnostic accuracy of US, MRI and MRA in the characterisation of RC full-thickness tears, meta-analysis showed a similar performance of the three imaging modalities for overall estimates of $\mathrm{Sn}(0.90$ to 0.91$)$ and $\mathrm{Sp}(0.93$ to 0.95 ; table $2 \mathrm{~A}$, figure 4). Figure 5 illustrates the HSROC curves of the US, MRI and MRA in the characterisation of full-thickness RC tears.

Secondary analysis regarding risk of bias evaluation

Grouping studies according to the risk of bias on patient selection did not reveal any significant difference for US. For MRI (Sn: 0.83 and 0.90, respectively) and MRA (Sn: 0.83 and 0.93, respectively), Sn was slightly lower for the low risk of bias 
Table 2 Overall Sn and Sp of US, MRI and MRA in the characterisation of full-thickness rotator cuff tears

\begin{tabular}{|c|c|c|c|c|c|c|}
\hline & \multicolumn{6}{|c|}{ (A) Rotator cuff full-thickness tear } \\
\hline & $\mathrm{N}$ (studies) & N (shoulders) & Sn (Cl 95\%) & $\mathrm{Sp}(\mathrm{Cl} 95 \%)$ & LR+ & LR- \\
\hline US & $30^{\mathrm{a}}$ & 2402 & $0.91(0.86$ to 0.94$)$ & 0.93 (0.91 to 0.96$)$ & 13.0 & 0.1 \\
\hline MRI & $23^{b}$ & 1581 & $0.90(0.85$ to 0.95$)$ & 0.93 (0.89 to 0.97$)$ & 12.9 & 0.1 \\
\hline MRA & $14^{c}$ & 1544 & $0.90(0.83$ to 0.95$)$ & 0.95 (0.91 to 0.98$)$ & 18.0 & 0.1 \\
\hline
\end{tabular}

(B) Secondary analysis based on risk of bias

(B) Secondary analysis based on risk of bias
Studies with low risk of bias in patient selection

\begin{tabular}{|c|c|c|c|c|c|c|c|c|c|c|c|c|}
\hline & 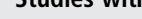 & ( & & & & & \multicolumn{6}{|c|}{ 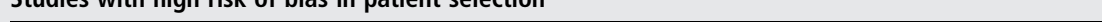 } \\
\hline & N (studies) & N (shoulders) & Sn (Cl 95\%) & Sp (CI 95\%) & $\mathrm{LR}+$ & LR- & N (studies) & N (shoulders) & Sn (Cl 95\%) & $\mathrm{Sp}(\mathrm{Cl} 95 \%)$ & LR+ & LR- \\
\hline US & $8^{\mathrm{d}}$ & 587 & $0.87(0.72$ to 0.97$)$ & 0.94 (0.88 to 0.99$)$ & 14.5 & 0.1 & $23^{9}$ & 1825 & 0.90 (0.85 to 0.94 ) & 0.93 (0.90 to 0.97 ) & 12.9 & 0.1 \\
\hline MRI & $7^{e}$ & 484 & $0.83(0.75$ to 0.95$)$ & 0.91 (0.80 to 0.97$)$ & 9.2 & 0.2 & $13^{\mathrm{h}}$ & 759 & 0.90 (0.80 to 0.97 ) & 0.93 (0.86 to 0.98 ) & 12.9 & 0.2 \\
\hline MRA & $8^{f}$ & 1181 & $0.83(0.68$ to 0.93$)$ & $0.96(0.91$ to 1$)$ & 20.8 & 0.2 & $6^{\mathrm{i}}$ & 295 & 0.93 (0.86 to 0.98$)$ & $0.90(0.82$ to 0.96$)$ & 9.3 & 0.1 \\
\hline
\end{tabular}

(C) Secondary analysis based on equipment characteristics

$\leq 7.5 \mathrm{MHz}$ (US), $\leq 1.5 \mathrm{~T}$ (MRI and MRA) $\quad>7.5 \mathrm{MHz}$ (US), 3.0 T (MRI and MRA)

\begin{tabular}{|c|c|c|c|c|c|c|c|c|c|c|c|c|}
\hline & \multicolumn{6}{|c|}{ 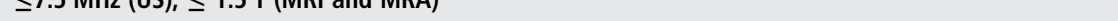 } & \\
\hline & N (studies) & N (shoulders) & Sn (CI 95\%) & Sp (Cl 95\%) & $\mathrm{LR}+$ & LR- & N (studies) & N (shoulders) & Sn (Cl 95\%) & Sp (CI 95\%) & LR+ & LR- \\
\hline US & $17^{\mathrm{j}}$ & 1535 & 0.88 (0.80 to 0.94$)$ & 0.94 (0.89 to 0.97$)$ & 14.7 & 0.1 & $13^{m}$ & 910 & $0.90(0.82$ to 0.96$)$ & $0.93(0.88$ to 0.97$)$ & 12.9 & 0.1 \\
\hline MRI & $21^{\mathrm{k}}$ & 1238 & $0.90(0.86$ to 0.95$)$ & $0.92(0.88$ to 0.96$)$ & 11.3 & 0.1 & $2^{n}$ & 198 & $0.89(0.54$ to 1$)$ & 0.89 (0.59 to 1$)$ & 8.1 & 0.1 \\
\hline MRA & $10^{1}$ & 1299 & 0.87 (0.78 to 0.94$)$ & 0.95 (0.90 to 0.99$)$ & 17.4 & 0.1 & $3^{\circ}$ & 177 & 0.90 (0.63 to 1$)$ & 0.90 (0.68 to 1$)$ & 9.0 & 0.1 \\
\hline
\end{tabular}

(D) Secondary analysis based on the specialist who performed the US examination

Radiologists $\quad$ Sonographers and orthopaedists

\begin{tabular}{|c|c|c|c|c|c|c|c|c|c|c|c|c|}
\hline & \multicolumn{6}{|l|}{ Radiologists } & \multicolumn{6}{|c|}{ Sonographers and orthopaedists } \\
\hline & $\mathrm{N}$ (studies) & N (shoulders) & Sn (CI 95\%) & $\mathrm{Sp}(\mathrm{Cl} 95 \%)$ & LR+ & LR- & $\mathrm{N}$ (studies) & N (shoulders) & Sn (CI 95\%) & Sp (CI 95\%) & LR+ & LR- \\
\hline US & $18^{p}$ & 1185 & 0.89 (0.82 to 0.94$)$ & 0.93 (0.89 to 0.97$)$ & 12.7 & 0.1 & $10^{q}$ & 916 & 0.86 (0.75 to 0.94$)$ & 0.94 (0.88 to 0.98$)$ & 14.3 & 0.1 \\
\hline
\end{tabular}

Included studies: (a) Refs 1922 27-29 $3133353739404244-4850537980-88$ (b) Refs $42831475456-6063656668-70808284899091$ (c) Refs $294257-5969727375767892-94$

(d) Refs 1931353639444682 (e) Refs 31576668698290 (f) Ref5 57697375789294 (g) Refs 22 27-29 33 37 40 42 45 47 $48505379-8183-88$ (c)

(l) Refs ${ }^{2} 575869737678929394$ (m) Refs $22 \quad 2933353637404780818688$ (n) Refs ${ }^{60} 90$ (o) Refs 297275 (p) Refs $192227293137404244464780-8488$ (q) Refs $2835-37394548537985$.

LR-, negative likelihood ratio; LR+, positive likelihood ratio; MRA, MR arthrography; $n$, number of studies or shoulders; Sn, sensitivity; Sp, specificity; US, ultrasonography. 
Figure 4 Accuracy of individual studies for US (A), MRI (B) and MRA (C) in characterization of full thickness rotator cuff tears. TP, true positive; $\mathrm{FP}$, false positive; FN, false negative; TN, true negative; $95 \% \mathrm{Cl}, 95 \%$ confidence interval.

\section{A Ultrasonography}

Study
Brandt 1989
Brenneke 1992
Chang 2001
Chang 2001
Cullen 2007
De Candia 2002
Hodler 1988
lannotti 2005
Kang 2009
Martin-Henas 2001
Milosavjlevic 2005
Moosmayer 2007
Murphy 2013
Naqvi 2009
Paavolainen 1994
Read 1998
Roberts 2001
Rutten 2010
Sipola 2010
Sonnabend 1997
Swen 1998
Swen 1999
Taboury 1992
Takagishi 1996
Teefey 2000
Teefey 2004
Van Holsbeek 1995
Venu 2002
Weiner 1993
Zeigler 2004

TP FP FN TN Sensitivity $(95 \% \mathrm{Cl})$ Specificity $(95 \% \mathrm{Cl})$ Sensitivity $(95 \% \mathrm{Cl})$ Specificity $(95 \% \mathrm{Cl})$ $\begin{array}{llllll}12 & 4 & 9 & 13 & 0.57[0.34,0.78] & 0.76[0.50,0.93]\end{array}$ $\begin{array}{llllll}36 & 6 & 2 & 76 & 0.95[0.82,0.99] & 0.93[0.85,0.97]\end{array}$ $\begin{array}{lllll}23 & 0 & 2 & 7 & 0.92[0.74,0.99]\end{array}$ $\begin{array}{lllll}16 & 1 & 15 & 11 & 0.52[0.33,0.70]\end{array}$ $\begin{array}{lllll}25 & 0 & 3 & 37 & 0.89[0.72,0.98]\end{array}$ $\begin{array}{lllll}32 & 0 & 1 & 38 & 0.97[0.84,1.00] \\ 35 & 1 & 0 & 15 & 1.00[0.90,1.00]\end{array}$ $\begin{array}{rrrrr}35 & 1 & 0 & 15 & 1.00[0.90,1.00] \\ 37 & 10 & 5 & 47 & 0.88[0.74,0.96]\end{array}$ $\begin{array}{rrrrr}37 & 10 & 5 & 47 & 0.88[0.74,0.96] \\ 35 & 1 & 5 & 9 & 0.88[0.73,0.96]\end{array}$ $\begin{array}{rrrrr}35 & 1 & 5 & 9 & 0.88[0.73,0.96]\end{array}$ $\begin{array}{rrrrr}15 & 0 & 11 & 35 & 0.58[0.37,0.77] \\ 94 & 9 & 0 & 87 & 100[0.96,100]\end{array}$ $\begin{array}{lllll}24 & 1 & 0 & 32 & 1.00[0.86,1.00]\end{array}$ $\begin{array}{lllll}37 & 12 & 3 & 67 & 0.93[0.80,0.98]\end{array}$ $\begin{array}{rrrrr}15 & 2 & 2 & 17 & 0.88[0.64,0.99]\end{array}$ $\begin{array}{lllll}15 & 2 & 2 & 17 & 0.88[0.64,0.99]\end{array}$ $1010031 \quad 1.00[0.69,1.00]$ $\begin{array}{lllll}8 & 0 & 2 & 14 & 0.80[0.44,0.97]\end{array}$ $\begin{array}{lllll}21 & 3 & 1 & 43 & 0.95[0.77,1.00]\end{array}$ $\begin{array}{lllll}48 & 9 & 10 & 10 & 0.83[0.71,0.91]\end{array}$ $38 \quad 5 \quad 7 \quad 60 \quad 0.84[0.71,0.94]$ $19 \quad 3 \quad 3 \quad 23 \quad 0.86[0.65,0.97]$ $\begin{array}{lllll}10 & 1 & 3 & 7 & 0.77[0.46,0.95\end{array}$ $\begin{array}{lllll}20 & 0 & 1 & 3 & 0.95[0.76,1.00]\end{array}$ $29 \quad 0 \quad 9 \quad 84 \quad 0.76[0.60,0.89]$ $\begin{array}{lllll}65 & 3 & 0 & 32 & 1.00[0.94,1.00]\end{array}$ $\begin{array}{lllll}45 & 5 & 1 & 20 & 0.98[0.88,1.00]\end{array}$ $34 \quad 000033 \quad 1.00[0.90,1.00]$ $\begin{array}{lllll}10 & 0 & 2 & 29 & 0.83[0.52,0.98]\end{array}$ $84 \quad 1 \quad 6 \quad 134 \quad 0.93[0.86,0.98]$ $1.00[0.59,1.00]$ $0.92[0.62,1.00]$ $1.00[0.91,1.00]$ $1.00[0.91,1.00]$ $0.94[0.70,1.00]$ $0.82[0.70,0.91]$ $0.90[0.55,1.00]$ $1.00[0.90,1.00]$ $0.91[0.83,0.96]$ $0.97[0.84,1.00]$ $0.85[0.75,0.92]$ $0.89[0.67,0.99]$ $0.95[0.77,1.00]$ $0.97[0.84,1.00]$ $1.00[0.77,1.00]$ $0.93[0.82,0.99]$ $0.53[0.29,0.76]$ $0.92[0.83,0.97]$ $0.88[0.70,0.98]$ $0.88[0.47,1.00]$ $1.00[0.29,1.00]$ $1.00[0.96,1.00]$ $0.91[0.77,0.98]$ $0.80[0.59,0.93]$ $1.00[0.89,1.00]$ $1.00[0.88,1.00]$ $0.99[0.96,1.00]$ $0.94[0.89,0.97]$

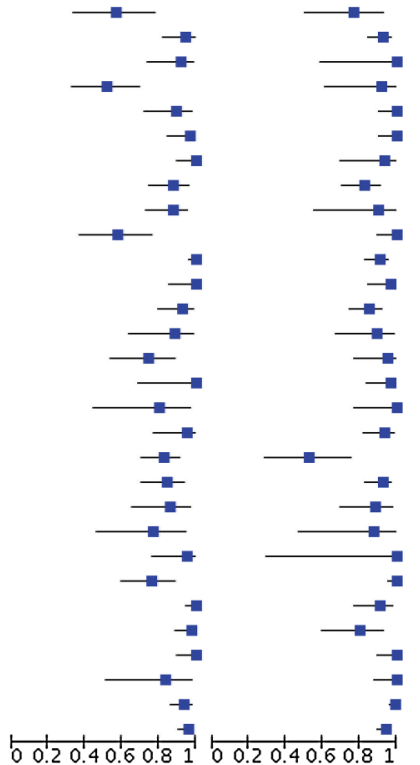

$B$ Magnetic resonance imaging

\begin{tabular}{|c|c|c|c|c|c|c|c|c|}
\hline Study & TP & FP & FN & TN & Sensitivity $(95 \% \mathrm{Cl})$ & Specificity $(95 \% \mathrm{Cl})$ & Sensitivity $(95 \% \mathrm{Cl})$ & Specificity $(95 \% \mathrm{Cl})$ \\
\hline Adams 2010 & 16 & 0 & 28 & 46 & $0.36[0.22,0.52]$ & $1.00[0.92,1.00]$ & $\rightarrow$ & - \\
\hline Balich 1997 & 41 & 6 & 4 & 171 & $0.91[0.79,0.98 j$ & $0.97[0.93,0.99]$ & $\rightarrow$ & - \\
\hline Blanchard 1999 & 9 & 6 & 2 & 21 & $0.82[0.48,0.98]$ & $0.78[0.58,0.91]$ & 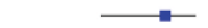 & $=-$ \\
\hline Chang 2002 & 27 & 0 & 4 & 12 & $0.87[0.70,0.96]$ & $1.00[0.74,1.00]$ & $\rightarrow-$ & $\longrightarrow$ \\
\hline Chang 2002 & 24 & 1 & 1 & 6 & $0.96[0.80,1.00]$ & $0.86[0.42,1.00]$ & $\rightarrow$ & -- \\
\hline Gagey 1993 & 14 & 1 & 0 & 23 & $1.00[0.77,1.00]$ & $0.96[0.79,1.00]$ & $\longrightarrow$ & $\longrightarrow$ \\
\hline Hitachi 2011 & 35 & 0 & 5 & 28 & $0.88[0.73,0.96]$ & $1.00[0.88,1.00]$ & $\rightarrow-$ & $\rightarrow$ \\
\hline Hodler 1992 & 4 & 4 & 0 & 28 & $1.00[0.40,1.00]$ & $0.88[0.71,0.96]$ & $\longrightarrow$ & $\rightarrow-$ \\
\hline Iannotti 1991 & 33 & 3 & 0 & 52 & $1.00[0.89,1.00]$ & $0.95[0.85,0.99]$ & $\rightarrow$ & $\rightarrow$ \\
\hline lannotti 2005 & 40 & 7 & 2 & 50 & $0.95[0.84,0.99]$ & $0.88[0.76,0.95]$ & $\rightarrow$ & $\rightarrow$ \\
\hline Karzel 1993 & 10 & 4 & 2 & 52 & $0.83[0.52,0.98]$ & $0.93[0.83,0.98]$ & $\rightarrow-$ & $\rightarrow$ \\
\hline Lambert 2009 & 32 & 0 & 1 & 15 & $0.97[0.84,1.00]$ & $1.00[0.78,1.00]$ & $\rightarrow$ & $\longrightarrow$ \\
\hline Magee 2006 & 96 & 2 & 2 & 50 & $0.98[0.93,1.00]$ & $0.96[0.87,1.00]$ & $\rightarrow$ & $\rightarrow$ \\
\hline Martin-Henas 2001 & 21 & 1 & 5 & 34 & $0.81[0.61,0.93]$ & $0.97[0.85,1.00]$ & $\rightarrow-$ & $\rightarrow$ \\
\hline $\mathrm{Nagvi} 2009$ & 33 & 3 & 3 & 16 & $0.92[0.78,0.98]$ & $0.84[0.60,0.97]$ & $\rightarrow$ & - \\
\hline Quinn 1995 & 17 & 1 & 3 & 79 & $0.85[0.62,0.97]$ & $0.99[0.93,1.00]$ & 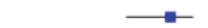 & $=$ \\
\hline Swen 1999 & 10 & 1 & 3 & 7 & $0.77[0.46,0.95]$ & $0.88[0.47,1.00]$ & $\rightarrow-$ & $\rightarrow-$ \\
\hline Teefey 2004 & 46 & 8 & 0 & 17 & $1.00[0.92,1.00]$ & $0.68[0.46,0.85]$ & $\rightarrow$ & \\
\hline Traughber 1992 & 5 & 0 & 0 & 23 & $1.00[0.48,1.00]$ & $1.00[0.85,1.00]$ & $\longrightarrow$ & $\rightarrow$ \\
\hline Tuite 1994 & 20 & 3 & 2 & 62 & $0.91[0.71,0.99]$ & $0.95[0.87,0.99]$ & $\longrightarrow$ & $\rightarrow$ \\
\hline Wnorowski 1997 & 6 & 7 & 3 & 23 & $0.67[0.30,0.93 \mid$ & $0.77[0.58,0.90]$ & & \\
\hline Yagci 2001 & 8 & 5 & 2 & 9 & $0.80|0.44,0.97|$ & $0.64[0.35,0.87]$ & $=-$ & \\
\hline Yamakawa 2001 & 46 & 1 & 8 & 20 & $0.85[0.73,0.93]$ & $0.95[0.76,1.00]$ & $\rightarrow$ & $\overrightarrow{\mathrm{t}}$ \\
\hline
\end{tabular}

C Magnetic resonance arthrography

\begin{tabular}{|c|c|c|c|c|c|c|c|c|}
\hline Study & TP & FP & $\mathbf{F N}$ & TN & Sensitivity $(95 \% \mathrm{Cl})$ & Specificity $(95 \% \mathrm{Cl})$ & Sensitivity $(95 \% \mathrm{Cl})$ & Specificity (95\% Cl) \\
\hline Binkert 2001 & 11 & 1 & 1 & 17 & $0.92[0.62,1.00]$ & $0.94[0.73,1.00]$ & & $\longrightarrow$ \\
\hline Choo 2012 & 16 & 2 & 1 & 30 & $0.94[0.71,1.00]$ & $0.94[0.79,0.99]$ & & $\rightarrow$ \\
\hline Herold 2006 & 17 & 0 & 3 & 31 & $0.85[0.62,0.97]$ & $1.00[0.89,1.00]$ & & $\rightarrow$ \\
\hline Hitachi 2011 & 11 & 0 & 12 & 16 & $0.48[0.27,0.69]$ & $1.00[0.79,1.00]$ & & $\longrightarrow$ \\
\hline Hodler 1992 & 4 & 4 & 0 & 28 & $1.00[0.40,1.00]$ & $0.88[0.71,0.96]$ & & $\rightarrow-$ \\
\hline Kang 2009 & 39 & 1 & 1 & 9 & $0.97[0.87,1.00]$ & $0.90[0.55,1.00]$ & $\rightarrow$ & $\longrightarrow$ \\
\hline Karzel 1993 & 12 & 4 & 0 & 52 & $1.00[0.74,1.00]$ & $0.93[0.83,0.98]$ & $\longrightarrow$ & $\rightarrow$ \\
\hline Loew 2000 & 12 & 0 & 0 & 15 & $1.00[0.74,1.00]$ & $1.00[0.78,1.00]$ & & \\
\hline Modi 2013 & 3 & 1 & 0 & 74 & $1.00[0.29,1.00]$ & $0.99[0.93,1.00]$ & & - \\
\hline Mohtadi 2004 & 10 & 4 & 0 & 44 & $1.00[0.69,1.00]$ & $0.92[0.80,0.98]$ & $\longrightarrow$ & $\rightarrow$ \\
\hline Sipola 2010 & 50 & 1 & 7 & 17 & $0.88[0.76,0.95]$ & $0.94[0.73,1.00]$ & $\rightarrow$ & \\
\hline Stoppino 2013 & 6 & 1 & 0 & 101 & $1.00[0.54,1.00]$ & $0.99[0.95,1.00]$ & & $=$ \\
\hline Waldt 2007 & 88 & 10 & 4 & 723 & $0.96[0.89,0.99]$ & $0.99[0.98,0.99]$ & $\rightarrow$ & \\
\hline Yagci 2001 & 10 & 0 & 0 & 14 & $1.00[0.69,1.00]$ & $1.00[0.77,1.00]$ & & $\longrightarrow$ \\
\hline
\end{tabular}

group of studies (table 2B). The credible intervals of the Sn, however, did overlap.

Secondary analysis regarding equipment characteristics

For characterisation of full-thickness RC tears, pooling data regarding US transducer frequency $(\leq 7.5 \mathrm{MHz}$ or $>7.5 \mathrm{MHz})$ did not show any significant difference (table 2C). Similarly, for the electromagnetic flux density of MRI and MRA, the differences of overall $\mathrm{Sn}$ and $\mathrm{Sp}$ between $\leq 1.5 \mathrm{~T}$ and
3.0 T were not statistically significant (credible intervals overlapped).

Secondary analysis regarding the specialist performing the US examination

The meta-analyses showed a similar diagnostic accuracy of US when the examination was performed by a trained radiologist or by other professionals (trained sonographers or orthopaedists; table 2D). 


\section{A Ultrasonography}
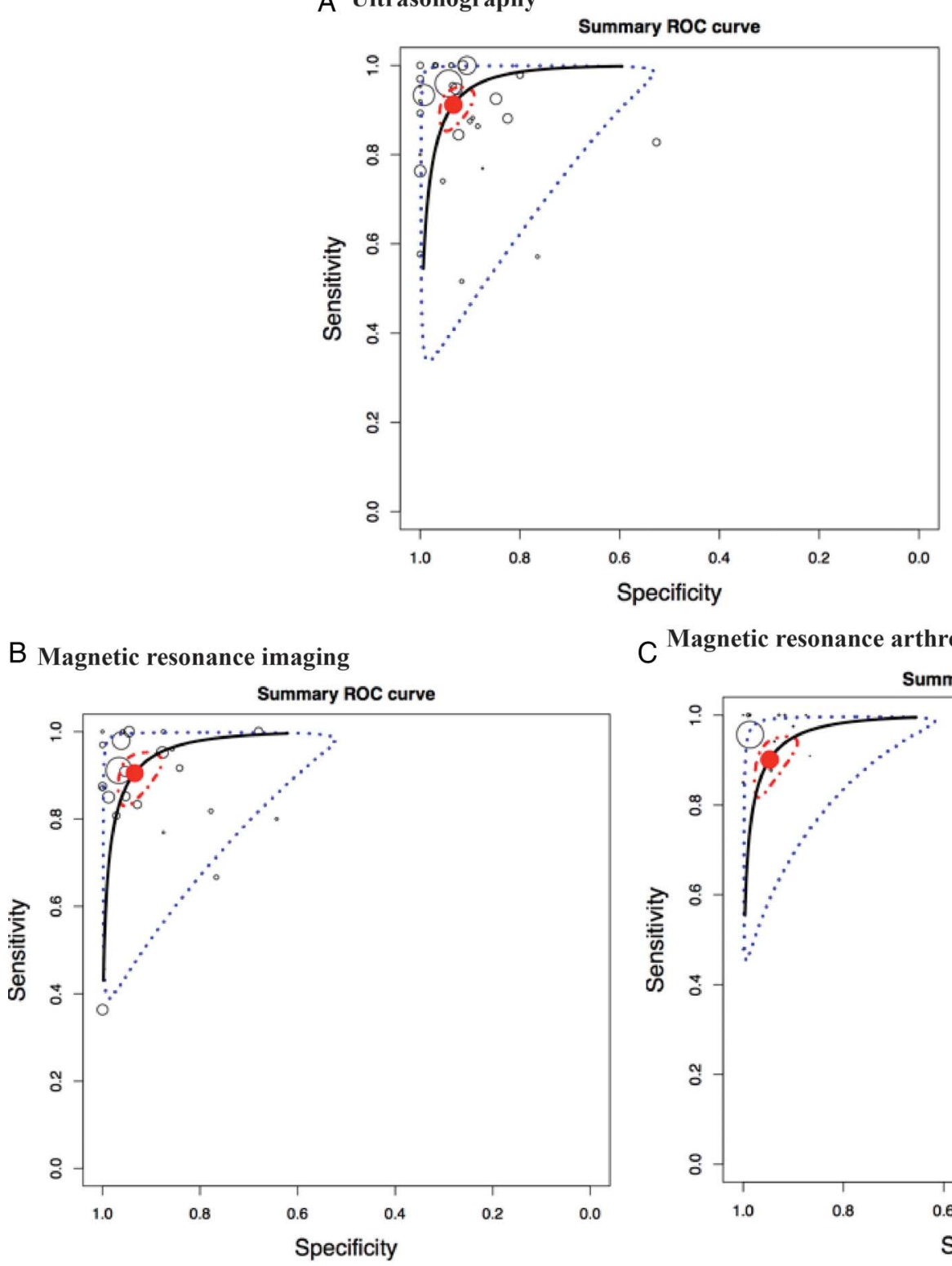

C Magnetic resonance arthrography

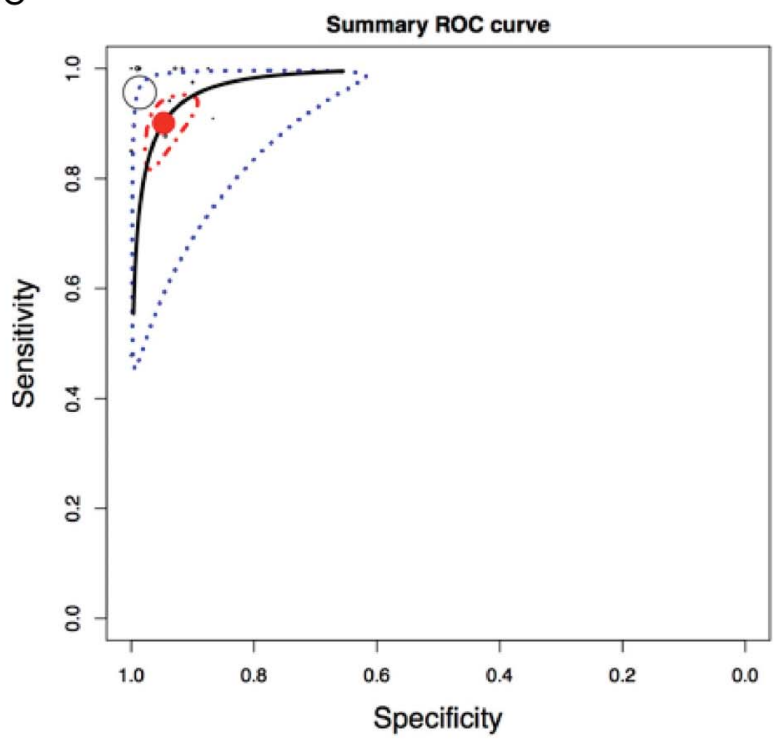

Figure 5 Hierarchical summary ROC curve for full thickness rotator cuff tears. Hierarchical summary receiver operating characteristic (HSROC) curve composed of studies examining the diagnostic value of the ultrasonography, MRI and MR arthrography in characterisation of full-thickness or partial-thickness rotator cuff tears. Circles represent each study, blue dotted line, the $\mathrm{Cl}$ and red dotted line the credible interval. TP, true positive; FP, false positive; FN, false negative; TN, true negative.

\section{Diagnostic accuracy of imaging modalities for partial thickness RC tear}

Pooled data for all included studies

For the diagnosis of partial RC tears, while the overall Sn of US and MRI were similar (0.68 and 0.67, respectively), that of MRA's was higher (Sn 0.83; table 3A, figure 6). For the overall estimate of $\mathrm{Sp}$, the three imaging modalities performed equivalently $(0.93-$ 0.94). Figure 7 illustrates the HSROC curves of the US, MRI and MRA in the characterisation of partial thickness RC tears.

\section{Secondary analysis regarding risk of bias evaluation}

The results of the secondary analysis regarding the risk of bias in patient selection revealed an important difference, particularly for US, between the group of studies with a high risk of bias and the group of studies with a low risk of bias for Sn ( 0.77 and 0.43 , respectively for US; table $3 \mathrm{~B})$. The intervals of the overall estimates of sensitivity for these groups, although quite large, barely overlapped. The overall estimate of Sp was 0.94 in both groups. With the MRI studies, similar overall estimates of $\mathrm{Sn}$ and $\mathrm{Sp}$ were found. As for MRA, the overall estimates of $\mathrm{Sn}$ were similar in both groups. A difference of $12 \%$ between the overall estimates of $\mathrm{Sp}$ were, however, observed, the estimate being higher in the group of studies with a low risk of bias (Sp: 0.97) than in the group of studies with a high risk of bias (Sp: 0.85). Again, the credible intervals of the overall estimates specificity for these groups barely overlapped.

\section{Secondary analysis of equipment characteristics}

For the diagnostic accuracy of US in characterisation of partial RC tears, there seems to be no advantage to use a frequency higher or lower than $7.5 \mathrm{MHz}$ (table 3C). For MRI, this comparison revealed non-significant differences of $12 \%$ in $\mathrm{Sn}$ and $8 \%$ in Sp between a density of $1.5 \mathrm{~T}$ and of $3.0 \mathrm{~T}$ in favour of 3.0 T. Based on likelihood ratios though, a $3.0 \mathrm{~T}$ density would 
Table 3 Overall Sn and Sp of US, MRI and MRA in the characterisation of partial thickness rotator cuff tears

\begin{tabular}{|c|c|c|c|c|c|c|c|c|c|c|c|c|}
\hline & & \multicolumn{11}{|c|}{ (A) Partial thickness rotator cuff tear } \\
\hline & & \multicolumn{2}{|l|}{ N (studies) } & N (shoulders) & \multicolumn{3}{|c|}{$\mathrm{Sn}(\mathrm{Cl} 95 \%)$} & \multicolumn{2}{|c|}{ Sp (Cl 95\%) } & \multicolumn{2}{|l|}{ LR+ } & LR- \\
\hline US & & \multicolumn{2}{|l|}{$23^{\mathrm{a}}$} & 2109 & \multicolumn{3}{|c|}{$0.68(0.54$ to 0.83$)$} & \multicolumn{2}{|c|}{0.94 (0.90 to 0.97$)$} & \multicolumn{2}{|l|}{11.3} & 0.3 \\
\hline MRI & & $16^{\mathrm{b}}$ & & 1195 & \multicolumn{3}{|c|}{$0.67(0.50$ to 0.82$)$} & \multicolumn{2}{|c|}{0.94 (0.88 to 0.99$)$} & \multicolumn{2}{|l|}{11.2} & 0.4 \\
\hline \multirow[t]{4}{*}{ MRA } & & $15^{c}$ & & 1885 & \multicolumn{3}{|c|}{$0.83(0.74$ to 0.91$)$} & \multicolumn{2}{|c|}{0.93 (0.88 to 0.98$)$} & \multicolumn{2}{|l|}{11.9} & 0.2 \\
\hline & \multicolumn{12}{|c|}{ (B) Secondary analysis based on risk of bias } \\
\hline & \multicolumn{6}{|c|}{ Studies with low risk of bias in patient selection } & \multicolumn{6}{|c|}{ Studies with high risk of bias in patient selection } \\
\hline & N (studies) & N (shoulders) & Sn (Cl 95\%) & $\mathrm{Sp}(\mathrm{Cl} 95 \%)$ & LR+ & LR- & N (studies) & N (shoulder) & Sn (Cl 95\%) & $\mathrm{Sp}(\mathrm{Cl} 95 \%)$ & LR+ & LR- \\
\hline US & $7^{d}$ & 492 & $0.43(0.16$ to 0.76 & 0.94 (0.84 to 1$)$ & 7.2 & 0.6 & $16^{9}$ & 1617 & $0.77(0.64$ to 0.89$)$ & 0.94 (0.89 to 0.97$)$ & 12.8 & 0.2 \\
\hline MRI & $5^{e}$ & 487 & $0.64(0.32$ to 0.88 & $0.95(0.80$ to 1$)$ & 12.8 & 0.4 & $8^{\mathrm{h}}$ & 495 & $0.70(0.44$ to 0.92$)$ & $0.92(0.80$ to 0.99$)$ & 8.8 & 0.3 \\
\hline \multirow[t]{4}{*}{ MRA } & $6^{f}$ & 1236 & 0.81 (0.64 to 0.92 & 0.97 (0.91 to 0.99$)$ & 27.0 & 0.2 & $8^{i}$ & 447 & $0.86(0.76$ to 0.95$)$ & $0.85(0.74$ to 0.93$)$ & 5.7 & 0.2 \\
\hline & \multicolumn{12}{|c|}{ (C) Secondary analysis based on equipment characteristics } \\
\hline & \multicolumn{6}{|c|}{$\leq 7.5 \mathrm{MHz}$ (US), $\leq 1.5 \mathrm{~T}$ (MRI and MRA) } & \multicolumn{6}{|c|}{ >7.5 MHz (US), 3.0 T (MRI and MRA) } \\
\hline & N (studies) & N (shoulders) & Sn (Cl 95\%) & $\mathrm{Sp}(\mathrm{Cl} 95 \%)$ & LR+ & LR- & N (studies) & N (shoulders) & Sn (Cl 95\%) & $\mathrm{Sp}(\mathrm{Cl} 95 \%)$ & $\mathbf{L R}+$ & LR- \\
\hline US & $12^{\mathrm{j}}$ & 1300 & 0.68 (0.49 to 0.85 & 0.94 (0.90 to 0.98 ) & 11.3 & 0.3 & $10^{\mathrm{m}}$ & 756 & 0.64 (0.38 to 0.88$)$ & $0.93(0.85$ to 0.99$)$ & 9.1 & 0.3 \\
\hline MRI & $13^{k}$ & 847 & $0.61(0.42$ to 0.80 & $0.90(0.82$ to 0.96$)$ & 6.1 & 0.4 & $3^{n}$ & 348 & $0.73(0.44$ to 0.97$)$ & $0.98(0.85$ to 1$)$ & 36.5 & 0.3 \\
\hline \multirow[t]{4}{*}{ MRA } & $10^{\prime}$ & 1504 & 0.82 (0.68 to 0.94 & $0.91(0.81$ to 0.97$)$ & 9.1 & 0.2 & $4^{0}$ & 303 & $0.85(0.63$ to 0.98$)$ & $0.93(0.81$ to 1$)$ & 12.1 & 0.2 \\
\hline & \multicolumn{12}{|c|}{ (D) Secondary analysis based on the specialist who performed the US examination } \\
\hline & \multicolumn{6}{|l|}{ Radiologists } & \multicolumn{6}{|c|}{ Sonographers and orthopaedists } \\
\hline & N (studies) & N (shoulders) & Sn (Cl 95\%) & Sp (Cl 95\%) & LR+ & LR- & N (studies) & N (shoulders) & Sn (Cl 95\%) & $\mathrm{Sp}(\mathrm{Cl}$ 95\%) & LR+ & LR- \\
\hline US & $14^{\mathrm{p}}$ & 1005 & 0.65 (0.44 to 0.85$)$ & 0.92 (0.86 to 0.98$)$ & 8.1 & 0.4 & $7^{9}$ & 748 & 0.64 (0.35 to 0.88$)$ & 0.94 (0.87 to 0.99 ) & 10.7 & 0.4 \\
\hline
\end{tabular}


Figure 6 Accuracy of individual studies for ultrasonography, MRI and MR arthrography in characterisation of full thickness rotator cuff tears.

TP, true positive; FP, false positive;

FN, false negative; TN, true negative.

\section{A Ultrasonography}

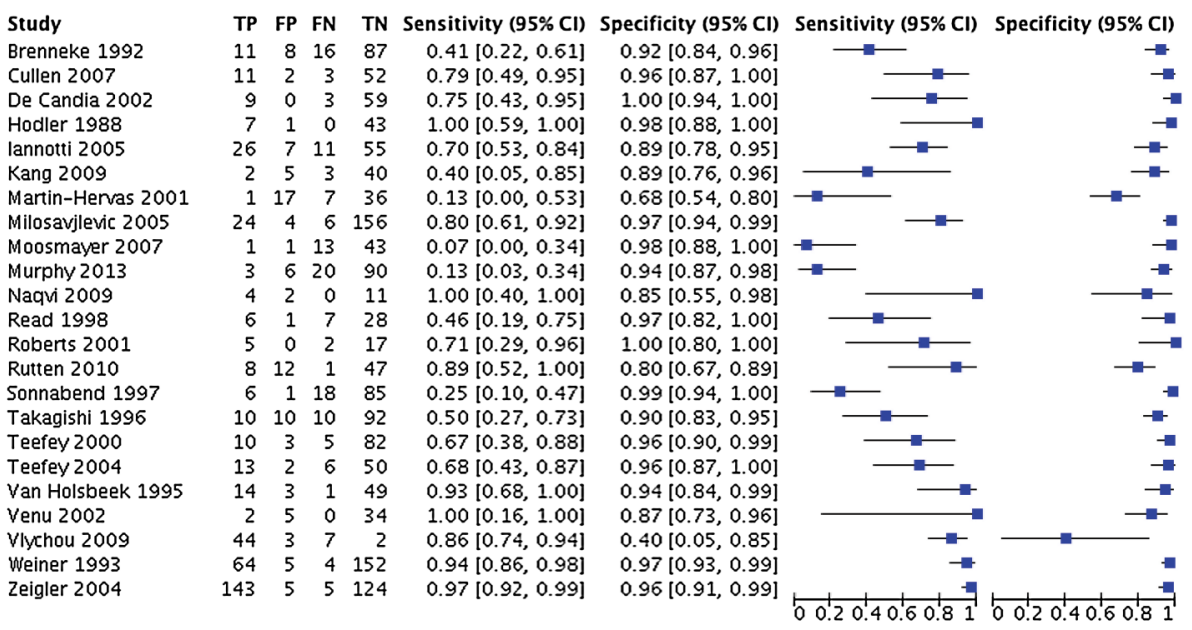

\section{$\mathrm{B}$ Magnetic resonance imaging}

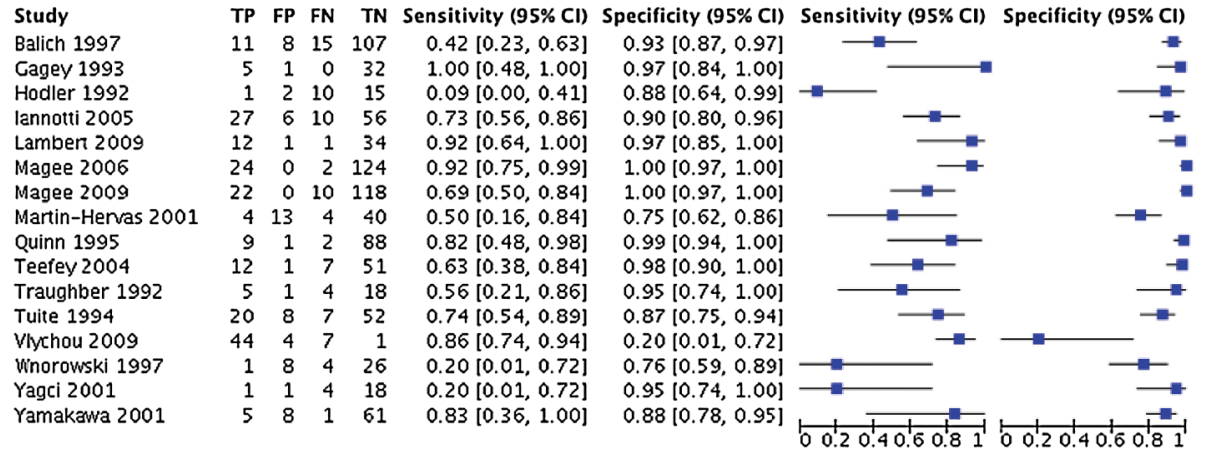

\section{Magnetic resonance arthrography}

\begin{tabular}{|c|c|c|c|c|c|c|c|c|c|}
\hline Study & TP & FP & FN & $\mathrm{TN}$ & Sensitivity $(95 \% \mathrm{Cl})$ & Specificity $(95 \% \mathrm{Cl})$ & Sensitivity $(95 \% \mathrm{CI})$ & Specificity (9 & $95 \% \mathrm{Cl})$ \\
\hline Choo 2012 & 15 & 4 & 3 & 27 & $0.83[0.59,0.96]$ & $0.87[0.70,0.96]$ & & & \\
\hline Chun 2010 & 9 & 2 & 11 & 180 & $0.45[0.23,0.68]$ & $0.99[0.96,1.00]$ & & & \\
\hline Herold 2006 & 11 & 3 & 3 & 34 & $0.79[0.49,0.95]$ & $0.92[0.78,0.98]$ & & & \\
\hline Jung 2010 & 21 & 4 & 3 & 16 & $0.88[0.68,0.97]$ & $0.80[0.56,0.94]$ & & & \\
\hline Jung 2012 & 26 & 2 & 2 & 24 & $0.93[0.76,0.99]$ & $0.92[0.75,0.99]$ & & & \\
\hline Kang 2009 & 4 & 2 & 1 & 43 & $0.80[0.28,0.99]$ & $0.96[0.85,0.99]$ & & & \\
\hline Magee 2009 & 31 & 0 & 1 & 118 & $0.97[0.84,1.00]$ & $1.00[0.97,1.00]$ & $\rightarrow$ & & \\
\hline Meister 2004 & 26 & 5 & 2 & 34 & $0.93[0.76,0.99]$ & $0.87[0.73,0.96]$ & & & \\
\hline Modi 2013 & 5 & 1 & 4 & 68 & $0.56[0.21,0.86]$ & $0.99[0.92,1.00]$ & $\Rightarrow$ & & \\
\hline Mohtadi 2004 & 16 & 17 & 10 & 15 & $0.62[0.41,0.80]$ & $0.47[0.29,0.65]$ & $\longrightarrow-$ & $\longrightarrow$ & \\
\hline Sipola 2010 & 5 & 8 & 2 & 60 & $0.71[0.29,0.96]$ & $0.88[0.78,0.95]$ & & & \\
\hline Stetson 2005 & 21 & 4 & 2 & 23 & $0.91[0.72,0.99]$ & $0.85[0.66,0.96]$ & $\longrightarrow$ & & \\
\hline Stoppino 2013 & 27 & 1 & 3 & 77 & $0.90[0.73,0.98]$ & $0.99[0.93,1.00]$ & $\rightarrow$ & & \\
\hline Waldt 2007 & 84 & 24 & 21 & 696 & $0.80[0.71,0.87]$ & $0.97[0.95,0.98]$ & - & & \\
\hline Yagci 2001 & 5 & 2 & 0 & 17 & $1.00[0.48,1.00]$ & $0.89[0.67,0.99]$ & & & \\
\hline
\end{tabular}

be more efficient for the confirmation of a partial thickness RC tear, compared to a density of $1.5 \mathrm{~T}$. The same can be concluded for MRA, where overall estimates of $\mathrm{Sn}$ and $\mathrm{Sp}$ were similar, but likelihood ratios revealed a slight advantage in favour of a flux density of $3.0 \mathrm{~T}$.

Secondary analysis regarding the specialist performing the US examination

Here again, the meta-analyses showed a similar performance by a trained radiologist or by other professionals (trained sonographers or orthopaedists; table 3D).

\section{Diagnostic accuracy of imaging modalities for RC tendinopathy}

It was possible to combine five studies (311 shoulders; arthroscopy or open surgery as reference standard) that provided data for the diagnostic accuracy of US in the characterisation of tendinopathy. Overall estimate of $\mathrm{Sn}$ of this group of studies was 0.79 and the overall estimate of Sp was 0.94 (table 4).

\section{DISCUSSION}

This meta-analysis revealed very good diagnostic accuracy of US, MRI and MRA in the characterisation of full-thickness RC tears, while the overall sensitivity of these tools was lower for the characterisation of partial RC tears and tendinopathy (the later was only evaluated for US). For partial thickness RC tears, the overall sensitivity of MRA was found to be slightly higher than those of MRI and US. An electromagnetic flux density of 3.0 T was also found to confirm a partial thickness RC tear with more confidence than with a density of $1.5 \mathrm{~T}$ (albeit, the difference was not significant). However, the studies published on the diagnostic accuracy of flux density of $3.0 \mathrm{~T}$ were more recent (range: 2009 to 2013; mean: 2010) compared to the studies evaluating flux density of $1.5 \mathrm{~T}$ (range: 1991 to 2013; mean: 


\section{A Ultrasonography}

Summary ROC curve

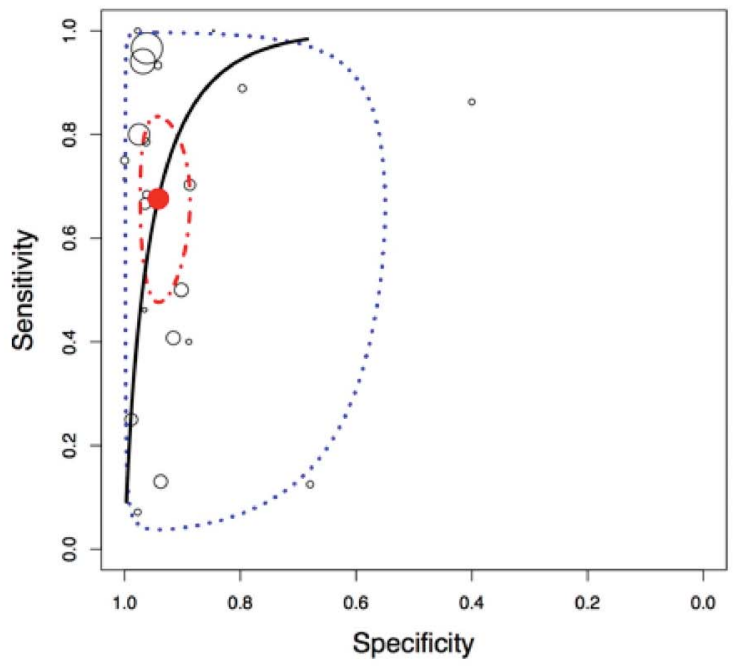

B Magnetic resonance imaging

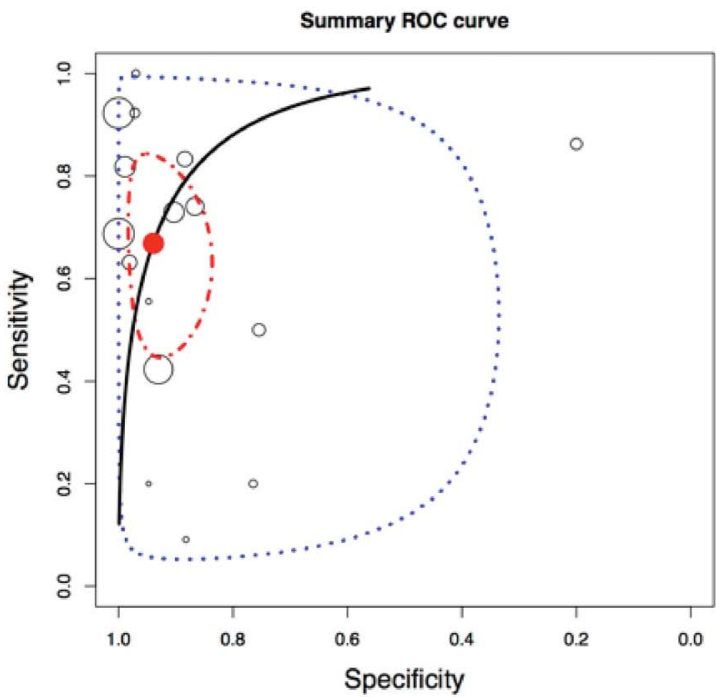

C Magnetic resonance arthrography

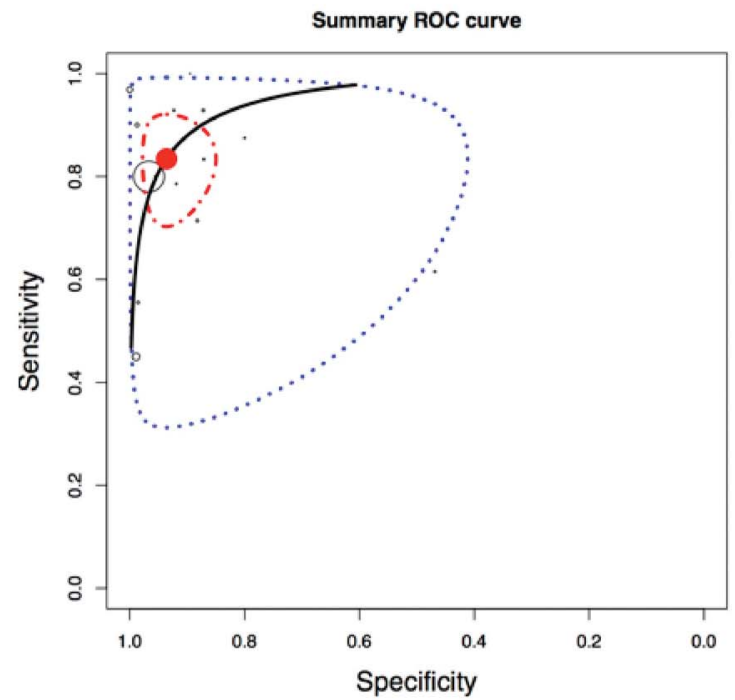

Figure 7 Hierarchical summary ROC curves for partial thickness rotator cuff tears. Hierarchical summary receiver operating characteristic (HSROC) curve composed of studies examining the diagnostic value of the ultrasonography (A), MRI (B) and MRA (C) in characterisation of full or partial thickness RC tears. Circles represent each study, blue dotted line, the confidence interval and red dotted line the credible interval.

2001). Therefore confounding factors such as improved technology also need to be considered when interpreting this result.

Finally, in cases of partial RC tears diagnosed by US, the overall sensitivity was lower in the group of studies with a low risk of bias in participant selection compared to the group of studies with a high risk of bias. This means that studies that have included participants with a large spectrum of shoulder conditions, and not only participants strongly suspected of having RC diseases, would have provided a more valid estimate of the sensitivity of US. It is likely that when a preselection of participants is made as to their probable diagnosis, the sensitivity of US is overestimated due to an increased prevalence of RC pathologies. ${ }^{100}$

\section{Comparison to other systematic reviews with meta-analysis} Our results are consistent with previous systematic reviews, ${ }^{5-7}$ 9-11 but add new data and evidence on the diagnostic accuracy. The most recent systematic review of imaging, published in $2013,{ }^{5}$ included only patients for whom surgery for a RC disorder had

Table 4 Overall Sn and Sp of US for the characterisation of tendinopathy

\begin{tabular}{|c|c|c|c|c|c|c|}
\hline & \multicolumn{6}{|c|}{ Tendinopathy } \\
\hline & N (studies) & $\mathrm{N}$ (shoulders) & Sn (Cl 95\%) & $\mathrm{Sp}(\mathrm{Cl} 95 \%)$ & LR+ & LR- \\
\hline US & $5^{a}$ & 311 & 0.79 (0.63 to 0.91$)$ & 0.94 (0.86 to 0.99$)$ & 13.2 & 0.2 \\
\hline
\end{tabular}


been considered. These highly specific eligibility criteria allowed inclusion of a limited number of studies, which could explain the differences in estimated diagnostic accuracy between that study and the present one. For example, the overall estimate of sensitivity for MRI in the characterisation of any RC tears was 0.98 and overall estimate of specificity was $0.79 .^{14}$ In our study, the equivalent estimates were 0.90 for sensitivity and specificity.

In two meta-analyses by Smith et al, ${ }^{10}{ }^{11}$ similar results as those found in the present study were obtained; however, for partial thickness RC tears for US, differences were noted. They obtained an overall estimate of sensitivity of 0.84 compared to 0.68 in the present study. This could be explained by the fact that some studies that were included in the aforementioned systematic reviews were excluded in the present review for several reasons: unable to reconstruct the $2 \times 2$ tables (seven articles), study population ineligible (three articles), not in English (four studies), surgery not the reference standard (two studies) and articles not on diagnostic accuracy (three articles). Further, 26 articles included in the present study were not included in the Smith et al's meta-analysis (mostly more recent studies). For partial thickness RC tears, our conclusions coincide more closely to those of De Jesus et $a l^{6}$ and Dinnes et al. $^{7}$ In these meta-analyses, despite the differences in the statistical methods used and in the number of included studies, the conclusions remain very close to ours. In accordance with their conclusions, we also found equivalent performance between the three diagnostic tools, with a slight advantage to MRA for partial thickness RC tears. Partial $\mathrm{RC}$ tears are the most difficult to detect, but the specificity of all tools was high even for this specific pathology.

The consistency of findings across systematic reviews with different search strategies, inclusion criteria and dates covered confirms the high specificity of US, MRI and MRA. Conversely, the pooled estimate of sensitivity varies among the reviews and our findings represent the most recent, rigorous estimate of overall accuracy and factors that might contribute to variation.

\section{Clinical implications}

From our findings, US, MRI and MRA can be considered highly specific tools for the diagnosis of RC disorders, while being highly sensitive mostly for full-thickness RC tears. Beside diagnostic value, several factors must be considered to appreciate the clinical implications of such results. Safety, cost, availability and impact of the results of clinical management are key elements. Regarding safety, beside some specific contraindications for MRI, US and MRI are non-invasive tests. Claustrophobia can be an issue with MRI and MRA. Although MRA showed a slightly better performance, this procedure involves an intra-articular injection that can cause inconveniences to patients (discomfort, risk of infection). When considering cost and availability, these tests can be classified as follows in most settings: US < MRI < MRA. ${ }^{101} 102$ Finally, and likely most important when requesting imaging for $\mathrm{RC}$ injuries, one must consider the impact of the test results on clinical management.

In general, partial RC tears are treated using modalities similar to tendinopathy while RC repair surgery is considered in cases of full-thickness RC tears associated with a number of criteria such as duration (acute vs chronic), age, function, pain and size of tear. ${ }^{103}$ The most clinically important characteristic when selecting a shoulder imaging modality for RC disorder evaluation is therefore the capacity to properly detect a full-thickness RC tear since it constitutes a key indication for surgical repair. ${ }^{104}$ Hence, the most clinically relevant aspect of this meta-analysis is the data related to the capacity of the tests to document full-thickness RC tears.
Interestingly, one finding of this study is that, when performed by specially trained operators, shoulder US may not be as user dependent as previously thought since, for the diagnosis of a full-thickness RC tear, diagnosis was similar whether trained radiologists, sonographers or orthopaedists performed the US examination. However, it is important to take into consideration that the sonographers and orthopaedists who performed the US examination in the included articles were specially trained, and that there is a steep learning curve to US. ${ }^{14}$ Therefore, the use of ultrasound at the point of care must be linked to specific training that should be defined in curriculum such as the one recently updated by the American Medical Society for Sports Medicine (AMSSM). ${ }^{105}$ Finally, diagnostic use of US at the point of care can also lead to improved therapeutic efficiency through the immediate use of interventions such as injections, which have been shown to be more effective under US guidance. ${ }^{106} 107108$

The use of US, providing equivalent information to MRI but less expensive, is therefore recommended for the diagnosis of RC disorders. In the event that US or MRI do not provide the diagnosis, MRA may be indicated. These conclusions are consistent with the imaging algorithms for evaluating suspected RC disease proposed by the Society of Radiologists in Ultrasound. ${ }^{102}$ Also, clinical situations where other shoulder conditions such as articular cartilage injuries or labral tears must be considered (eg, in cases where glenohumeral instability in younger patients or osteoarthritis in older patients overlap with RC disorders) could justify the use of MRI or MRA. However, in most conditions, first we advocate a combination of different non-invasive and less expensive clinical evaluation tests before the use of medical imaging, as these tests are mostly appropriate for confirming a number of specific RC pathologies. ${ }^{108}$ However, in situations of acute shoulder injuries where fullthickness RC tears is considered, imaging must be obtained rapidly as rapid repair of a 'fresh' tear on a previously healthy $\mathrm{RC}$ has the best prognosis.

\section{Strengths and limitations}

This meta-analysis was based on a rigorous literature search, which resulted in the inclusion of 82 articles, and a validated appraisal tool was used to determine the risk of bias of included studies. Still, there are some limitations. With the statistical package used in the present study, we were able to calculate confidence and credible intervals for the overall sensitivity and specificity, but not for the likelihood ratios. No method was found to calculate CIs around the likelihood ratios that are derived from overall estimates of sensitivity and specificity. Other limitations include that 47 studies were specifically excluded because of incomplete data reporting (unable to construct a $2 \times 2$ table). There were also recurrent sources of bias on three of the four items of the QUADAS 2 tool, which shows poor reporting of participants' characteristics and study design for the included studies. Finally, while the two raters were fairly concordant when evaluating the risk of bias $(\kappa>0.50)$, one item was less concordant (item 1: patient selection; $\kappa=0.33$ ). However, agreement was easily obtained between to two raters and the initial disagreements were mostly related to adherence to the defined standard.

\section{SUMMARY}

The high and stable specificity across studies of the US, MRI and MRA leads us to recommend their uses for the confirmation of RC pathologies in patients already suspected of having those problems on the basis of clinical evaluation tests in acute cases 
where surgery might be considered rapidly or in situations where conservative treatments have failed. When considering accuracy, cost, availability, safety and efficiency of management when used at the point of care, US is likely be the best option in most settings for the diagnosis of full-thickness RC tears.

\section{What are the new findings}

- The meta-analysis confirms the similar and high diagnostic accuracy of ultrasonography (US), MRI and MR arthrography (MRA) in the characterisation of full-thickness rotator cuff (RC) tears in individuals with shoulder pain.

- Conversely, the pooled estimate of sensitivity varies among the reviews and our findings represent the most recent, rigorous estimate of overall accuracy and factors that might contribute to variation. Thus, the sensitivity of the US, MRI and MRA is more susceptible to variation for all diagnoses of RC disorders, while the specificity of these diagnostic tools is stable and high.

- Diagnostic accuracy of US is similar whether a trained radiologist, sonographer or orthopaedist performed the US examination.

\section{Author affiliations}

'Department of Rehabilitation, Faculty of Medicine, Université Laval, Quebec City, Quebec, Canada

${ }^{2}$ Center for Interdisciplinary Research in Rehabilitation and Social Integration, Quebec City, Quebec, Canada

${ }^{3}$ Maisonneuve-Rosemont Hospital Research Center, University of Montreal Affiliated Research Center, Montreal, Quebec, Canada

${ }^{4}$ Faculty of Medicine, School of Rehabilitation, Universite de Montreal, Montreal, Quebec, Canada

${ }^{5}$ Centre de recherche du Centre Hospitalier Universitaire de Québec, Quebec City, Quebec, Canada

${ }^{6}$ School of Rehabilitation Science, McMaster University, Hamilton, Ontario, Canada ${ }^{7}$ Department of Radiology, Research Center, Centre hospitalier de I'Université de Montréal, Montreal, Quebec, Canada

Contributors J-SR, FD, CED, JCM, NB and PF participated in the design of the study. J-SR, CB, JL, FD, CED, JCM, NB and PF participated in the analysis and the interpretation of data and drafted the manuscript. J-SR and CB carried out the acquisition of data and the evaluation of the risk of bias. JL is the statistician.

Funding Financial support has been provided by the Institut de Recherche RobertSauvé en Santé et Sécurité au Travail (IRSST) and the Réseau Provincial de Recherche en Adaptation-Réadaptation/Fonds de recherche du Québec - Santé (REPAR/FRQ-S). JS Roy was supported by a salary award from the FRQ-S. JC MacDermid was supported by a Chair in Gender, Work and Health by the Canadian Institutes of Health Research.

\section{Competing interests None.}

Provenance and peer review Not commissioned; externally peer reviewed.

Open Access This is an Open Access article distributed in accordance with the Creative Commons Attribution Non Commercial (CC BY-NC 4.0) license, which permits others to distribute, remix, adapt, build upon this work non-commercially, and license their derivative works on different terms, provided the original work is properly cited and the use is non-commercial. See: http://creativecommons.org/ licenses/by-nc/4.0/

\section{REFERENCES}

1 van der Heijden GJ. Shoulder disorders: a state-of-the-art review. Baillieres Clin Rheumatol 1999;13:287-309.

2 van der Windt DA, Koes BW, Boeke AJ, et al. Shoulder disorders in general practice: prognostic indicators of outcome. Br J Gen Pract 1996:46:519-23.

3 Neer CS. Impingement lesions. Clin Orthop Relat Res 1983;(173):70-7.

4 Blanchard TK, Bearcroft PW, Constant CR, et al. Diagnostic and therapeutic impact of MRI and arthrography in the investigation of full-thickness rotator cuff tears. Eur Radiol 1999;9:638-42.
5 Lenza M, Buchbinder R, Takwoingi Y, et al. Magnetic resonance imaging, magnetic resonance arthrography and ultrasonography for assessing rotator cuff tears in people with shoulder pain for whom surgery is being considered. Cochrane Database Syst Rev 2013;9:CD009020.

6 De Jesus JO, Parker L, Frangos AJ, et al. Accuracy of MRI, MR arthrography, and ultrasound in the diagnosis of rotator cuff tears: a meta-analysis. $A J R A m J$ Roentgenol 2009;192:1701-7.

7 Dinnes J, Loveman E, Mclntyre L, et al. The effectiveness of diagnostic tests for the assessment of shoulder pain due to soft tissue disorders: a systematic review. Health Technol Assess 2003;7:1-166.

8 Kelly AM, Fessell D. Ultrasound compared with magnetic resonance imaging for the diagnosis of rotator cuff tears: a critically appraised topic. Semin Roentgenol 2009;44:196-200.

9 Ottenheijm RP, Jansen MJ, Staal JB, et al. Accuracy of diagnostic ultrasound in patients with suspected subacromial disorders: a systematic review and meta-analysis. Arch Phys Med Rehabil 2010;91:1616-25.

10 Smith TO, Back T, Toms AP, et al. Diagnostic accuracy of ultrasound for rotator cuff tears in adults: a systematic review and meta-analysis. Clin Radiol 2011;66:1036-48.

11 Smith TO, Daniell H, Geere JA, et al. The diagnostic accuracy of MRI for the detection of partial- and full-thickness rotator cuff tears in adults. Magn Reson Imaging 2012;30:336-46.

12 Leeflang MM. Systematic reviews and meta-analyses of diagnostic test accuracy. Clin Microbiol Infect 2014;20:105-13.

13 Coris EE, Pescasio M, Zwygart K, et al. Office-based ultrasound in sports medicine practice. Clin I Sport Med 2011;21:57-61.

14 Harmon KG, O'Connor FG. Musculoskeletal ultrasound: taking sports medicine to the next level. Br I Sports Med 2010;44:1135-6.

15 Liberati A, Altman DG, Tetzlaff J, et al. The PRISMA statement for reporting systematic reviews and meta-analyses of studies that evaluate healthcare interventions: explanation and elaboration. BMJ 2009;339:b2700.

16 Whiting PF, Rutjes AW, Westwood ME, et al. QUADAS-2: a revised tool for the quality assessment of diagnostic accuracy studies. Ann Intern Med 2011;155:529-36.

17 Alqunaee M, Galvin R, Fahey T. Diagnostic accuracy of clinical tests for subacromial impingement syndrome: a systematic review and meta-analysis. Arch Phys Med Rehabil 2012:93:229-36.

18 Trikalinos TA, Balion CM, Coleman $\mathrm{Cl}$, et al. Meta-analysis of test performance when there is a "gold standard." AHRQ Publication No. 12-EHC080-EF. In: Chang SM, Matchar DB, Smetana GW, et al eds. Methods guide for medical test reviews. Agency for healthcare research and quality (US); 2012. Chapter 8:8-1 to 8-17.

19 Brenneke SL, Morgan CJ. Evaluation of ultrasonography as a diagnostic technique in the assessment of rotator cuff tendon tears. Am J Sports Med 1992;20:287-9.

20 Bretzke CA, Crass JR, Craig EV, et al. Ultrasonography of the rotatory cuff. Normal and pathologic anatomy. Invest Radiol 1985;20:311-15.

21 Chiou H, Chou Y, Wu J, et al. High-resolution ultrasonography of the musculoskeletal system: analysis of 369 cases. J Med Ultrasound 1999;7:212-18.

22 De Candia A, Doratiotto S, Pelizzo F, et al. Real time compound ultrasound of the shoulder. Radiol Oncol 2002;36:319-325+336.

23 De Muynck M. Sonography in the diagnosis of rotator cuff ruptures. Eur J Phys Rehab Med 1994;4:130-3.

24 Farin $\mathrm{P}$, Jaroma $\mathrm{H}$. Sonographic detection of tears of the anterior portion of the rotator cuff (subscapularis tendon tears). J Ultrasound Med 1996;15:221-5.

25 Friedman RL, Hidalgo HJ, Gilmer PW, et al. Ultrasonography of the rotator cuff: analysis of results in a community setting. I Shoulder Elbow Surg 1993;2:22-6.

26 Furtschegger $\mathrm{A}$, Resch $\mathrm{H}$. Value of ultrasonography in preoperative diagnosis of rotator cuff tears and postoperative follow-up. Eur J Radiol 1988:8:69-75.

27 Hodler J, Fretz CJ, Terrier F, et al. Rotator cuff tears: correlation of sonographic and surgical findings. Radiology 1988;169:791-4.

28 lannotti JP, Ciccone J, Buss DD, et al. Accuracy of office-based ultrasonography of the shoulder for the diagnosis of rotator cuff tears. J Bone Joint Surg Am 2005;87:1305-11.

29 Kang $\mathrm{CH}$, Kim SS, Kim JH, et al. Supraspinatus tendon tears: Comparison of 3D US and MR arthrography with surgical correlation. Skeletal Radiol 2009;38:1063-9.

30 Mack LA, Gannon MK, Kilcoyne RF, et al. Sonographic evaluation of the rotator cuff. Accuracy in patients without prior surgery. Clin Orthop Relat Res 1988 (234):21-7.

31 Martin-Hervas C, Romero J, Navas-Acien A, et al. Ultrasonographic and magnetic resonance images of rotator cuff lesions compared with arthroscopy or open surgery findings. J Shoulder Elbow Surg 2001;10:410-15.

32 Masaoka $\mathrm{S}$, Hashizume $\mathrm{H}$, Senda $\mathrm{M}$, et al. Ultrasonographic analysis of shoulder rotator cuff tears. Acta Medica Okayama 1999;53:81-9.

33 Milosavljevic J, Elvin A, Rahme H. Ultrasonography of the rotator cuff: a comparison with arthroscopy in one-hundred-and-ninety consecutive cases. Acta Radio 2005;46:858-65.

34 Misamore GW, Woodward C. Evaluation of degenerative lesions of the rotator cuff. A comparison of arthrography and ultrasonography. J Bone Joint Surg Am $1991 ; 73: 704-6$ 
35 Moosmayer S, Heir S, Smith HJ. Sonography of the rotator cuff in painful shoulders performed without knowledge of clinical information: Results from 58 sonographic examinations with surgical correlation. J Clin Ultrasound 2007;35:20-6.

36 Moosmayer S, Smith HJ. Diagnostic ultrasound of the shoulder-a method for experts only? Results from an orthopedic surgeon with relative inexpensive compared to operative findings. Acta Orthop 2005;76:503-8.

37 Murphy RJ, Daines MT, Carr AJ, et al. An independent learning method for orthopaedic surgeons performing shoulder ultrasound to identify full-thickness tears of the rotator cuff. J Bone Joint Surg Am 2013;95:266-72.

38 Pattee GA, Snyder SJ. Sonographic evaluation of the rotator cuff: correlation with arthroscopy. Arthroscopy 1988:4:15-20.

39 Roberts CS, Walker JA, II, Seligson D. Diagnostic capabilities of shoulder ultrasonography in the detection of complete and partial rotator cuff tears. Am J Orthop 2001;30:159-62.

40 Rutten MJ, Spaargaren GJ, Van Loon T, et al. Detection of rotator cuff tears: the value of MRI following ultrasound. Eur Radiol 2010;20:450-7.

41 Singh H, Yuvarajan P, Maini L, et al. Evaluation of ultrasound as a tool for etiological diagnosis of painful arc syndrome. J Clin Orthop Trauma 2010;1:81-4.

42 Sipola $\mathrm{P}$, Niemitukia L, Kroger $\mathrm{H}$, et al. Detection and quantification of rotator cuff tears with ultrasonography and magnetic resonance imaging - a prospective study in 77 consecutive patients with a surgical reference. Ultrasound Med Biol 2010;36:1981-9.

43 Soble MG, Kaye AD, Guay RC. Rotator cuff tear: clinical experience with sonographic detection. Radiology 1989;173:319-21.

44 Sonnabend DH, Hughes JS, Giuffre BM, et al. The clinical role of shoulder ultrasound. Aust NZ J Surg 1997;67:630-3.

45 Takagishi K, Makino K, Takahira N, et al. Ultrasonography for diagnosis of rotator cuff tear. Skeletal Radiol 1996:25:221-4.

46 Teefey SA, Hasan SA, Middleton WD, et al. Ultrasonography of the rotator cuff. A comparison of ultrasonographic and arthroscopic findings in one hundred consecutive cases. J Bone Joint Surg Am 2000;82:498-504.

47 Teefey SA, Rubin DA, Middleton WD, et al. Detection and quantification of rotator cuff tears. Comparison of ultrasonographic, magnetic resonance imaging, and arthroscopic findings in seventy-one consecutive cases. J Bone Joint Surg Am 2004;86-A:708-16.

48 Van Holsbeeck MT, Kolowich PA, Eyler WR, et al. US depiction of partial-thickness tear of the rotator cuff. Radiology 1995;197:443-6.

49 Wallny TA, Schild RL, Bertelsbeck DS, et al. Three-dimensional ultrasonography in the diagnosis of rotator cuff lesions. Ultrasound Med Biol 2001;27:745-9.

50 Wiener SN, Seitz Jr WH. Sonography of the shoulder in patients with tears of the rotator cuff: accuracy and value for selecting surgical options. AJR Am J Roentgenol 1993;160:103-7.

51 Yen $\mathrm{CH}$, Chiou HJ, Chou YH, et al. Six surgery-correlated sonographic signs for rotator cuff tears: Emphasis on partial-thickness tear. Clin Imaging 2004;28:69-76.

52 Zehetgruber $\mathrm{H}$, Lang T, Wurnig C. Distinction between supraspinatus, infraspinatus and subscapularis tendon tears with ultrasound in 332 surgically confirmed cases. Ultrasound Med Biol 2002;28:711-17.

53 Ziegler DW. The use of in-office, orthopaedist-performed ultrasound of the shoulder to evaluate and manage rotator cuff disorders. I Shoulder Elbow Surg 2004; 13:291-7

54 Balich SM, Sheley RC, Brown TR, et al. MR imaging of the rotator cuff tendon: interobserver agreement and analysis of interpretive errors. Radiology 1997;204:191-4

55 Frei R, Chladek P, Trc T, et al. Arthroscopic evaluation of ultrasonography and magnetic resonance imaging for diagnosis of rotator cuff tear. Ortop Traumatol Rehabil 2008;10:111-14.

56 Gagey N, Desmoineaux P, Gagey 0, et al. Apport de l'IRM dans le bilan pré-chirurgical des lésions de la coiffe des rotateurs. Revue de chirurgie orthopédique et réparatrice de l'appareil moteur 1991;77:521-9.

57 Hitachi S, Takase K, Tanaka M, et al. High-resolution magnetic resonance imaging of rotator cuff tears using a microscopy coil: noninvasive detection without intraarticular contrast material. Jpn J Radiol 2011;29:466-74.

58 Hodler J, Kursunoglu-Brahme S, Snyder SJ, et al. Rotator cuff disease: assessment with MR arthrography versus standard MR imaging in 36 patients with arthroscopic confirmation. Radiology 1992;182:431-6.

59 Karzel RP, Snyder SJ. Magnetic resonance arthrography of the shoulder: a new technique of shoulder imaging. Clin Sports Med 1993;12:123-36.

60 Lambert A, Loffroy R, Guiu B, et al. [Rotator cuff tears: value of 3.0T MRI] J Radiol 2009:90:583-8.

61 Magee T. 3-T MRI of the shoulder: Is MR arthrography necessary? AJR Am J Roentgenol 2009;192:86-92.

62 Ostor AJ, Richards CA, Tytherleigh-Strong G, et al. Validation of clinical examination versus magnetic resonance imaging and arthroscopy for the detection of rotator cuff lesions. Clin Rheumatol 2013;32:1283-91.

63 Quinn SF, Sheley RC, Demlow TA, et al. Rotator cuff tendon tears: evaluation with fat-suppressed MR imaging with arthroscopic correlation in 100 patients. Radiology 1995;195:497-500.
64 Shellock FG, Bert JM, Fritts HM, et al. Evaluation of the rotator cuff and glenoid labrum using a 0.2-Tesla extremity magnetic resonance (MR) system: MR results compared to surgical findings. J Magn Reson Imaging 2001;14:763-70.

65 Traughber PD, Goodwin TE. Shoulder MRI: arthroscopic correlation with emphasis on partial tears. J Comput Assist Tomogr 1992;16:129-33.

66 Tuite MJ, Yandow DR, DeSmet AA, et al. Diagnosis of partial and complete rotator cuff tears using combined gradient echo and spin echo imaging. Skeletal Radiol 1994:23:541-5.

67 Wang YM, Shih TT, Jiang CC, et al. Magnetic resonance imaging of rotator cuff lesions. J Formos Med Assoc 1994;93:234-9.

68 Wnorowski DC, Levinsohn EM, Chamberlain BC, et al. Magnetic resonance imaging assessment of the rotator cuff: Is it really accurate? Arthroscopy 1997:13:710-9.

69 Yagci B, Manisali M, Yilmaz E, et al. Indirect MR arthrography of the shoulder in detection of rotator cuff ruptures. Eur Radiol 2001;11:258-62

70 Yamakawa S, Hashizume H, Ichikawa N, et al. Comparative studies of $\mathrm{MRI}$ and operative findings in rotator cuff tear. Acta Med Okayama 2001;55:261-8

71 Zlatkin MB, Hoffman C, Shellock FG. Assessment of the rotator cuff and glenoid labrum using an extremity MR system: MR results compared to surgical findings from a multi-center study. J Magn Reson Imaging 2004;19:623-31.

$72 \mathrm{Choo} \mathrm{HJ}$, Lee SJ, $\mathrm{Kim} \mathrm{OH}$, et al. Comparison of three-dimensional isotropic T1-weighted fast spin-echo MR arthrography with two-dimensional MR arthrography of the shoulder. Radiology 2012;262:921-31.

73 Herold T, Bachthaler M, Hamer OW, et al. Indirect MR arthrography of the shoulder: use of abduction and external rotation to detect full- and partial-thickness tears of the supraspinatus tendon. Radiology 2006;240:152-60.

74 Jung JY, Jee WH, Park MY, et al. Supraspinatus tendon tears at 3.0T shoulder MR arthrography: diagnosis with 3D isotropic turbo spin-echo SPACE sequence versus 2D conventional sequences. Skeletal Radiol 2012;41:1401-10.

75 Modi CS, Karthikeyan S, Marks A, et al. Accuracy of abduction-external rotation MRA versus standard MRA in the diagnosis of intra-articular shoulder pathology. Orthopedics 2013;36:e337-42.

76 Mohtadi NG, Vellet AD, Clark ML, et al. A prospective, double-blind comparison of magnetic resonance imaging and arthroscopy in the evaluation of patients presenting with shoulder pain. I Shoulder Elbow Surg 2004:13:258-65

77 Schreinemachers SA, Van Der Hulst VPM, Willems WJ, et al. Detection of partial-thickness supraspinatus tendon tears: Is a single direct MR arthrography series in ABER position as accurate as conventional MR arthrography? Skeletal Radiol 2009;38:967-75.

78 Stoppino LP, Ciuffreda P, Rossi M, et al. Lesions of the rotator cuff footprint: diagnostic performance of MR arthrography compared with arthroscopy. Musculoskelet Surg 2013:97(Suppl 2):S197-202.

79 Brandt TD, Cardone BW, Grant TH, et al. Rotator cuff sonography: a reassessment. Radiology 1989:173:323-7.

80 Chang CY, Wang SF, Chiou HJ, et al. Comparison of shoulder ultrasound and MR imaging in diagnosing full-thickness rotator cuff tears. Clin Imaging 2002;26:50-4

81 Cullen DM, Breidahl WH, Janes GC. Diagnostic accuracy of shoulder ultrasound performed by a single operator. Australas Radiol 2007;51:226-9.

82 Naqvi GA, Jadaan M, Harrington P. Accuracy of ultrasonography and magnetic resonance imaging for detection of full thickness rotator cuff tears. Int I Shoulder Surg 2009;3:94.

83 Paavolainen P, Ahovuo J. Ultrasonography and arthrography in the diagnosis of tears of the rotator cuff. J Bone Joint Surg Am 1994;76:335-40.

84 Swen WAA, Jacobs JWG, Algra PR, et al. Sonography and magnetic resonance imaging equivalent for the assessment of full-thickness rotator cuff tears. Arthritis Rheum 1999:42:2231-8.

85 Swen WAA, Jacobs JWG, Neve WC, et al. Is sonography performed by the rheumatologist as useful as arthrography executed by the radiologist for the assessment of full thickness rotator cuff tears? J Rheumatol 1998:25:1800-6.

86 Taboury J. [Ultrasonography of the tendons of the rotator cuffs of the shoulder]. Ann Radiol 1995:38:275-9.

87 Read JW, Perko M. Shoulder ultrasound: diagnostic accuracy for impingement syndrome, rotator cuff tear, and biceps tendon pathology. J Shoulder Elbow Surg 1998; 7:264-71

88 Venu K, Howlett D, Garikipati R, et al. Evaluation of the symptomatic supraspinatus tendon-a comparison of ultrasound and arthroscopy. Radiography 2002:8:235-40.

89 Adams CR, Schoolfield JD, Burkhart SS. Accuracy of preoperative magnetic resonance imaging in predicting a subscapularis tendon tear based on arthroscopy. Arthroscopy 2010;26:1427-33.

90 Magee T, Williams D. 3.0-T MRI of the supraspinatus tendon. AMR Am J Roentgenol 2006;187:881-6.

91 lannotti JP, Zlatkin MB, Esterhai JL, et al. Magnetic resonance imaging of the shoulder. Sensitivity, specificity, and predictive value. J Bone Joint Surg Am 1991;73:17-29. 
92 Binkert CA, Zanetti M, Gerber C, et al. MR arthrography of the glenohumeral joint: two concentrations of gadoteridol versus ringer solution as the intraarticular contrast material. Radiology 2001;220:219-24.

93 Loew R, Kreitner KF, Runkel M, et al. MR arthrography of the shoulder: comparison of low-field (0.2T) vs high-field (1.5T) imaging. Eur Radiol 2000;10:989-96.

94 Waldt S, Bruegel M, Mueller D, et al. Rotator cuff tears: assessment with MR arthrography in 275 patients with arthroscopic correlation. Eur Radiol 2007;17:491-8.

95 Vlychou M, Dailiana Z, Fotiadou A, et al. Symptomatic partial rotator cuff tears: diagnostic performance of ultrasound and magnetic resonance imaging with surgical correlation. Acta Radiol 2009;50:101-5.

96 Chun KA, Kim MS, Kim YJ. Comparisons of the various partial-thickness rotator cuff tears on MR arthrography and arthroscopic correlation. Korean I Radiol 2010;11:528-35

97 Jung JY, Jee WH, Chun HJ, et al. Magnetic resonance arthrography including ABER view in diagnosing partial-thickness tears of the rotator cuff: accuracy, and inter- and intra-observer agreements. Acta Radiol 2010;51:194-201.

98 Meister K, Thesing J, Montgomery WJ, et al. MR arthrography of partial thickness tears of the undersurface of the rotator cuff: an arthroscopic correlation. Skeletal Radiol 2004;33:136-41.

99 Stetson WB, Phillips T, Deutsch A. The use of magnetic resonance arthrography to detect partial-thickness rotator cuff tears. J Bone Joint Surg Am 2005;87(Suppl 2):81-8.

100 Whiting P, Rutjes AW, Reitsma JB, et al. Sources of variation and bias in studies of diagnostic accuracy: a systematic review. Ann Intern Med 2004;140:189-202.
101 Parker L, Nazarian LN, Carrino JA, et al. Musculoskeletal imaging: medicare use, costs, and potential for cost substitution. J Am Coll Radiol 2008;5:182-8.

102 Nazarian LN, Jacobson JA, Benson CB, et al. Imaging algorithms for evaluating suspected rotator cuff disease: Society of Radiologists in Ultrasound consensus conference statement. Radiology 2013;267:589-95.

103 Marx RG, Koulouvaris P, Chu SK, et al. Indications for surgery in clinical outcome studies of rotator cuff repair. Clin Orthop Relat Res 2009;467: 450-6.

104 Lambers Heerspink FO, Dorrestijn O, van Raay JJ, et al. Specific patient-related prognostic factors for rotator cuff repair: a systematic review. I Shoulder Elbow Surg 2014;23:1073-80.

105 Finnoff J, Lavallee ME, Smith J. Musculoskeletal ultrasound education for sports medicine fellows: a suggested/potential curriculum by the American Medical Society for Sports Medicine. British I Sports Med 2010;44:1144-8.

106 Finnoff JT, Hall MM, Adams E, et al. American Medical Society for Sports Medicine (AMSSM) position statement: interventional musculoskeletal ultrasound in sports medicine. Br J Sports Med 2015;49:145-50.

107 Aly AR, Rajasekaran S, Ashworth N. Ultrasound-guided shoulder girdle injections are more accurate and more effective than landmark-guided injections: a systematic review and meta-analysis. Br J Sports Med 2015;49:1042-9.

108 Lasbleiz S, Quintero N, Ea K, et al. Diagnostic value of clinical tests for degenerative rotator cuff disease in medical practice. Ann Phys Rehabil Med 2014;57:228-43. doi:10.1016/j.rehab.2014.04.001 